\title{
Effects of unilateral tDCS over left prefrontal cortex on emotion regulation in depression: Evidence from concurrent functional magnetic resonance imaging
}

\author{
Wessel O. van Dam ${ }^{1} \cdot$ Evangelia G. Chrysikou ${ }^{1}$ \\ Accepted: 3 September 2020 / Published online: 12 January 2021 \\ (C) The Psychonomic Society, Inc. 2021
}

\begin{abstract}
Major depressive disorder (MDD) is the most common mood disorder and a primary cause of disability worldwide. MDD symptomatology entails disturbances in emotion regulation, namely one's ability to modify the intensity and duration of emotional reactions towards affective events. Transcranial direct current stimulation (tDCS) has emerged as a promising treatment for MDD. Yet, positive tDCS outcomes vary across studies, while the precise effects of the procedure for cortical excitability in MDD during emotion regulation remain largely unexplored. Here, we leveraged functional magnetic resonance imaging (fMRI)compatible tDCS technology to examine the functional consequences of a unilateral anodal tDCS montage at $1.5 \mathrm{~mA}$ over left PFC (area F3; with the reference electrode over an extracephalic location) for brain activity during an emotion-regulation task in MDD patients and age-matched healthy control subjects. Our results revealed down-regulation of negative emotions in the right amygdala and visual cortex of healthy controls but not MDD patients prior to stimulation, the degree of which correlated with the magnitude of the participants' reappraisal scores. TDCS did not elicit significant changes in neural activation patterns for either group. These findings contribute to the literature on the pathophysiology of MDD by showing that a key disturbance in the disorder entails the ineffective down-regulation of activity not only within the amygdala, but also within visual cortical areas in response to negative information. Further, these results suggest that relative to bifrontal tDCS montages, unilateral stimulation of moderate intensity over left PFC may not be sufficient to elicit therapeutic effects for MDD.
\end{abstract}

Keywords Transcranial direct current stimulation $\cdot$ Major depressive disorder $\cdot$ Emotion regulation $\cdot$ Visual cortex $\cdot$ Amygdala $\cdot$ Functional connectivity

\section{Introduction}

Major depressive disorder (MDD) is a serious, common, and frequently chronic condition that ranks among the primary causes of disability worldwide (Kessler, 2012; Kessler \& Bromet, 2013; World Health Organization, 2018). The primary symptoms of MDD involve pervasive sadness, loss of interest in activities once found enjoyable, and a marked difficulty in emotion regulation (ER). ER is generally defined as the set of both strategic and automatic processes that influence

Supplementary Information The online version of this article (https:// doi.org/10.3758/s13415-020-00830-4) contains supplementary material, which is available to authorized users.

Evangelia G. Chrysikou

lilachrysikou@drexel.edu

1 Department of Psychology, Drexel University, 3201 Chestnut St., Philadelphia, PA 19140, USA the occurrence, magnitude, duration, and expression of an affective event (John \& Gross, 2004; Gross \& Levenson, 1997). Individual differences guide the selection of particular emotion-regulation strategies, with cognitive reappraisal and expressive suppression identified as the most common among them (Gross \& John, 2003). Cognitive reappraisal refers to the strategy of re-interpreting an emotion-eliciting stimulus in a manner that changes the impact of its emotional response (Lazarus \& Alfert, 1964), whereas expressive suppression is an ER strategy that involves the inhibition of ongoing emotion-expressive behavior (Gross, 1998). It has been shown that cognitive reappraisal is associated with higher well-being and better interpersonal functioning, whereas expressive suppression is linked with experiencing and expressing lesser positive and greater negative emotion (Gross \& John, 2003; cf. Liverant, Brown, Barlow, \& Roemer, 2008). Examinations of the use of ER strategies in MDD have revealed, overall, less frequent or ineffective application of cognitive reappraisal relative to expressive suppression during ER 
(see Joormann \& Stanton, 2016, and Joormann \& Vanderlind, 2014, for reviews), which has been associated with increased depression symptoms (Joormann \& Gotlib, 2010) and rumination tendencies (Liverant, Kamholtz, Sloan, \& Brown, 2011) in MDD patients. Furthermore, expressive suppression seems to be applied similarly to the regulation of positive as well as negative affective responses in the disorder (Beblo et al., 2012).

The most common current treatments for addressing depressive symptomatology in MDD involve some combination of psychopharmacological and behavioral interventions. Unfortunately, these established treatments currently fail to elicit long-term and reliable therapeutic effects for an estimated $20-30 \%$ of MDD patients with the diagnosis (Rush et al., 2006). Alternative forms of antidepressant treatment have focused on different types of noninvasive brain stimulation, including repetitive transcranial magnetic stimulation (rTMS) and transcranial direct current stimulation (tDCS), with generally positive results across studies (Berlim et al., 2014; Janicak and Dokucu, 2015; Shiozawa et al., 2014). tDCS has emerged as a promising treatment for MDD with potentially broad impacts due to its ease of administration, safety profile, and low cost. The procedure involves the application of low-strength electrical currents (1-2.5 mA) by means of electrodes placed over the scalp for approximately 20-30 min (Bikson et al., 2016). Depending on polarity, anodal tDCS is presumed to result in neuron soma depolarization, thus generating excitatory effects on the cortex, whereas cathodal tDCS is presumed to result in neuron soma hyperpolarization, thus generating inhibitory effects on the cortex (Nitsche \& Paulus, 2000, 2001; Purpura \& McMurtry, 1965), although the exact excitability effects of tDCS on the brain are likely more complex (see Giordano et al., 2017; Jamil et al., 2017; Kronberg et al., 2017; Samani et al., 2019).

Due to the seemingly extensive network of cortical and subcortical regions - and their connectivity - implicated in MDD, the lower focality of tDCS (e.g., relative to rTMS) might present an advantage for its antidepressant efficacy, due to its potential to elicit widespread effects on the brain. The application of tDCS for the treatment of MDD has generally been implemented through medium-to-large $(5 \times 5 \mathrm{~cm}$ or $5 \times 7 \mathrm{~cm}$ ) electrodes and has involved various montages typically consisting of anodal electrode configurations over the left prefrontal cortex (PFC), with the cathode placed over the contralateral homologue region (Boggio et al., 2008; Fregni et al., 2006). Such electrode placements have been guided by a pathophysiological model of MDD that emphasizes the marked hypofunction - particularly during ER - in left dorsolateral and frontopolar PFC coupled with aberrant activity in ventrolateral, ventromedial, orbitofrontal, anterior cingulate, and subcortical regions (e.g., amygdala, hippocampus, basal ganglia; see DeRubeis et al., 2008; Disner et al., 2011, for reviews). Additional work has suggested a potential hemispheric imbalance in MDD with left PFC hypofunction typically accompanying right PFC hyperactivity, especially during negative ER tasks (Bush et al., 2000; Cowden Hindash \& Amir, 2012; Mitterschiffthaler et al., 2008). Consistent with this model, bifrontal tDCS montages with the anode over left PFC and the cathode over right PFC have mostly elicited reliable antidepressant effects relative to sham stimulation in small- and medium-scale controlled clinical trials (McClintock et al., 2020; see Brunoni et al., 2016, and Shiozawa et al., 2014, for reviews).

On the other hand, positive effects of tDCS have also been observed following unilateral montages, with the anode over left PFC and the cathode over an extracephalic location (see Arul-Anandam \& Loo, 2009; Fitzgerald et al., 2013; Martin et al., 2011). Indeed, electrical field modeling studies - currently applied routinely in a prescriptive manner to optimize electrode placement and ensure neural target engagement (e.g., Chase et al., 2019; Dmochowski et al., 2011) - have shown that montages involving the anode placed frontally with the reference electrode over an extracephalic location elicit more widespread activation (Bikson et al., 2008), whereas larger distance between the electrodes reduces current shunting across the scalp and consequently results in increased current inflow in the brain (Miranda et al., 2006). It is, thus, possible that unilateral montages might be more effective as treatment options for MDD patients. Nevertheless, the consistency and reliability of the potential antidepressant effects of bilateral versus unilateral prefrontal electrode montages for the treatment of MDD has not been systematically evaluated, and some variability remains across studies (Brunoni et al., 2016; Shiozawa et al., 2014).

A difficulty in assessing the relative efficacy of different tDCS montages for MDD, as well as the potential sources of variability of the observed results, stems from a persistent lack of understanding of the precise neurochemical and functional effects of tDCS on the brain across psychiatric disorders, including depression. This shortcoming can be significantly ameliorated through recent methodological advancements that have allowed the application of tDCS concurrently with functional magnetic resonance imaging (fMRI). However, only a handful of tDCS studies have used this multimodal imaging approach (e.g., Antal et al., 2011; Bergmann et al., 2016; Flöel, 2012; Hunter et al., 2013; Turi et al., 2012), despite its potential to elicit high-quality, artifact-free neuroimaging data reflective of online functional and neurochemical effects of tDCS on the brain (cf. Chrysikou et al., 2017; Meinzer et al., 2012; Sehm et al., 2012).

We have recently used fMRI-compatible tDCS technology to examine the precise neurofunctional consequences of tDCS for MDD during ER using a bifrontal montage (Chrysikou, Wing, \& van Dam, in press). Anodal tDCS was applied over left PFC (area F3 per the 10/20 system) together with cathodal tDCS over right PFC (F4) or sham tDCS during fMRI in 20 
patients with moderate-to-severe MDD and 20 gender- and age-matched control subjects. Participants performed two runs of an emotion-regulation (ER) task prior to tDCS and two runs of the task during tDCS, administered at $1.5-\mathrm{mA}$ with $5 \times$ $5 \mathrm{~cm}$ electrodes. Consistent with past literature (Lee et al., 2012; Ochsner et al., 2002, 2004; Phan et al., 2005), we found a deficient ER system in MDD involving ventromedial and orbitofrontal regions and the amygdala at baseline, which was more pronounced during reappraisal of negative stimuli (cf. Ochsner, Silvers, \& Buhle, 2012; Wager, Davidson, Hughes, Lindquist, \& Ochsner, 2008). Active anodal (but not sham) tDCS over DLPFC, with concurrent cathodal tDCS over right DLPFC, led to a transient reversal of this deviant pattern of activity in MDD patients relative to control subjects. These results provided the first evidence from a concurrent tDCSfMRI paradigm that a bifrontal tDCS montage alters cortical excitability in vivo during ER in depression, thus supporting the potential of this montage as antidepressant treatment.

Building on these results, here we used a similar paradigm to investigate the effect of unilateral anodal tDCS over left DLPFC with the cathode over the contralateral mastoid for ER of negative and positive stimuli in MDD patients and healthy control participants. Previous studies have demonstrated that reappraising negative stimuli modulates activity in the amygdala; that is, stronger activity in the amygdala is typically observed for a condition in which participants merely passively observe negative emotional pictures as compared to a condition in which they downregulate their emotional response to such pictures (Lee, Heller, van Reekum, Nelson \& Davidson, 2012; Ochsner et al., 2004; Phan et al., 2005). Findings with regard to the role of the amygdala in the processing of positive emotions have been less consistent, with evidence for both an increase and a decrease in activation of the left amygdala in response to happy faces (Breiter et al., 1996; Morris et al., 1996). Relative to negative stimuli, MDD patients' responses to positive stimuli have received considerably less attention, although differences in the processing of positive - and not only negative - emotional content might potentially underlie the pathophysiology of the disorder. For example, MDD patients have shown lower activity in the right anterior cingulate cortex and the left insula relative to healthy control subjects for positive stimuli, whereas depression severity appeared to relate to the processing of negative stimuli only (Lee et al., 2007). With regard to ER for positive emotional stimuli, Ochsner et al. (2012) reported that reappraisal of positive pictures does not seem to be consistently linked to modulation of activity in the amygdala in healthy participants. Despite the potential clinical significance of related findings for MDD, studies on the regulation of emotional processing for positive affective stimuli in mood disorders are scarce. The present study aimed to contribute to the literature on ER in MDD by including both negative and positive emotional content. In line with past literature, we anticipated that our wholebrain, region-of-interest, and functional connectivity analyses would reveal aberrant activity in ER networks including the amygdala and DLPFC in MDD patients at baseline for negative stimuli, which might be altered with unilateral tDCS (cf. Fitzgerald et al., 2013; Martin et al., 2011). Given inconsistencies in previous findings regarding the regulation of positive affect, we were sceptical about whether this pattern of results would hold for positive stimuli. No significant effects of tDCS were predicted for the healthy control participant group.

\section{Method}

\section{Participants}

Nineteen patients with major depressive disorder $\left(M_{\text {age }}=\right.$ 24.11 years, $S D=5.53$; two males; 11 receiving active stimulation) and 18 age-matched healthy control subjects $\left(M_{\text {age }}=23.94\right.$ years, $S D=4.57$, six males; 11 receiving active stimulation) participated in the study. Given the scarcity of combined tDCS-fMRI designs similar to the present study, the selection of sample size was based on an earlier study (Antal et al., 2011) that had employed the exact tDCS equipment and parameters in the MRI environment and obtained measurable tDCS effects with $\sim 10$ participants per group (with slightly lower stimulation at $1 \mathrm{~mA}$ than the $1.5 \mathrm{~mA}$ we employed in the present study). All participants were native English speakers by selfreport and had normal or corrected-to-normal vision. Participants further met all contraindications for MRI and tDCS testing (e.g., no metal implants; no claustrophobia; no history of head trauma or seizures; no current or suspected pregnancy as confirmed by a urine pregnancy test). Seven patients (37\%) were on low doses of antidepressant medications (two on sertraline, two on fluoxetine, and three on other related selective serotonin reuptake inhibitors [SSRIs]), four in the active tDCS condition and three in the sham tDCS condition. All participants signed informed consent and were paid for their time. The study was approved by the institutional review board at the University of Kansas Medical Center.

\section{Materials}

We used an adaptation of the Emotion Regulation Task (ERT), a well-established measure of ER, that has been associated with activation in PFC and the limbic system. The task consisted of 36 positive, 36 negative, and 18 neutral photographs from the International Affective Picture System (Lang, 
Bradley, \& Cuthbert, 2008). ${ }^{1}$ A two-tailed independent Student's $t$-test on the valence ratings indicated that negative pictures $(M=2.44, S D=1.50)$ had lower valence ratings than positive $(M=7.20, S D=1.58, t(35)=43.74, p<.00001)$ or neutral $(M=5.01, S D=1.21, t(35)=22.32, p<.00001)$ pictures. Positive pictures $(M=7.20)$ had higher valence ratings than neutral pictures $(M=5.01, t(35)=17.85, p<$ $.00001)$. A two-tailed independent Student's $t$-test on the arousal ratings indicated that negative pictures $(M=5.57, S D=2.14)$ had higher arousal ratings than positive $(M=4.72, S D=2.30$, $\mathrm{t}(35)=4.43, p<.0001)$ and neutral $(M=2.95, S D=1.84, t(35)$ $=10.27, p<.00001)$ pictures. Positive pictures $(M=4.72, S D=$ 2.30) had higher arousal ratings than neutral pictures $(M=2.95$, $S D=1.84, t(35)=9.88, p<.00001)$.

Half of all positive and negative pictures were preceded by instructions to decrease emotional responses using cognitive reappraisal. The other half were preceded by instructions to look at the picture and fully experience emotional responses. Assignment of the negative and positive pictures to the "Decrease" versus "Look" condition was counterbalanced across participants. Neutral pictures were preceded by instructions to look only. Thus, the experiment included the following five experimental conditions: (1) negative look (subject attended to a negative picture), (2) negative decrease (subject decreased their emotional response to a negative picture), (3) positive look (subject attended to a positive picture), (4) positive decrease (subject decreased emotional response to a positive picture), (5) neutral look (subject attended to a neutral picture). Following each trial, the participants were asked to rate their emotional response to each picture on a 4-point scale (for design, trial timing, and composition see Fig. 1). The task was implemented in E-Prime 2.0 (Psychology Software Tools, Pittsburgh, PA, USA) and split in four runs with durations of approximately $7 \mathrm{~min}$ each. Responses were made via button press through an MRI-compatible response box placed in the participants' dominant (right) hand.

\section{Procedure}

Participants first went through an introductory visit during which their depression status was evaluated using the BDIII; a clinician confirmed depression status and lack of comorbidity with other psychiatric disorders though the

\footnotetext{
${ }^{1}$ The IAPS numbers used for the negative pictures were the following (2053, 2095, 2141, 2205, 2276, 2301, 2455, 2457, 2683, 2691, 2700, 2703, 2750, $2800,2900,3220,3300,3500,3530,6212,6312,6313,6315,6350,6360$, $6520,6540,6550,6560,6561,6562,9041,9220,9331,9421,9921)$. The IAPS numbers used for the neutral pictures were the following $(2107,2200$, $2305,2495,2499,2512,2635,2840,7000,7006,7010,7040,7060,7090$, $7100,7150,7217,9070)$. The IAPS numbers for the positive pictures were the following $(1340,2035,2050,2154,2165,2304,2306,2340,2395,2550$, 4574, 7200, 7220, 7230, 7250, 7260, 7270, 7284, 7325, 7330, 7340, 7350, $7390,7400,7402,7405,7410,7430,7470,7480,8120,8330,8350,8380$, $8461,8540)$.
}

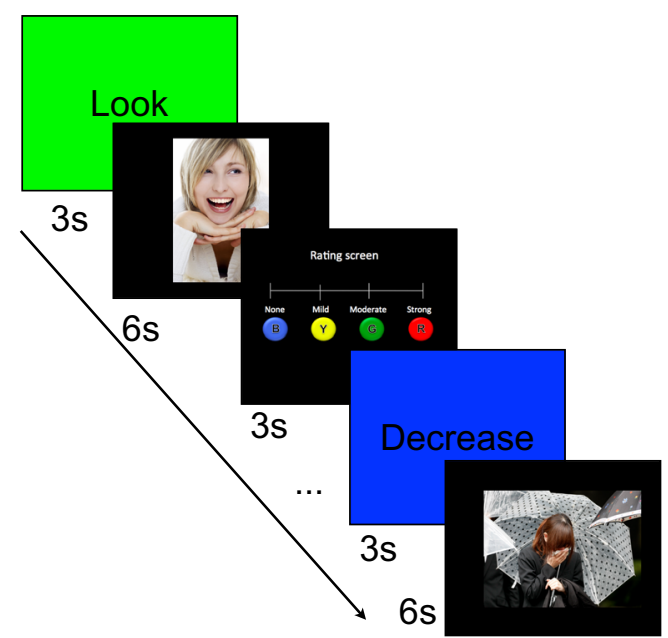

Fig. 1 Schematic overview of experimental trial by type. Colors on the emotional intensity rating scale indicate the color of the buttons on the MRI-compatible response box. $\mathrm{s}=$ seconds, $\mathrm{B}=$ Blue, $\mathrm{Y}=$ Yellow, $\mathrm{G}=$ Green, $\mathrm{R}=$ Red

Structural Clinical Interview for DSM-IV-II (SCID-II). Participants further received instructions and training on the experimental task. The introductory experimental session included exposure to tDCS that involved a description of the setup (i.e., identification of the locus of stimulation using a BraiNet cap and marking of that location), description of the stimulation, and the application of tDCS for approximately 3 min following the study montage. This exposure was done to ensure that participants were comfortable with the stimulation prior to scheduling their session at the brain-imaging center. It was long enough to allow them to experience what the stimulation feels like, but short enough to allow us to manipulate active versus sham administration during the main study unbeknownst to the subjects (all of whom experienced active stimulation during this exposure phase). The training phase included detailed instructions on the emotion-regulation task, adapted from the original paper that discussed it (Ochsner et al., 2004). Participants were told that the task they would complete in the scanner would involve the presentation of pictures that generally people assess as having positive, negative, or neutral valence. They were further told that each image would be preceded by instructions to either "Look" (which they were told means to look at the image that follows and naturally experience your emotional response to it), or "Decrease" (which they were told means to tell themselves something that would decrease their emotional response to the image [but avoid superficial strategies, like closing their eyes]). They were then told to anticipate a rating scale on which they were to indicate the intensity of their emotional response to the image after they had followed the instructions to either "Look" or "Decrease." Participants were given the opportunity to ask questions about these definitions and then they were shown five example images (two positive, two negative, and one neutral) that were not part of the main stimuli 
set used in the experiment. One of the positive and one of the negative images and the neutral image were preceded by the "Look" instructions; one of the positive and one of the negative images were preceded by the "Decrease" instructions. All images were followed by the rating scale. Each participant completed these five trials on the computer. Following these trials, we asked each participant to describe what strategies they followed to decrease their emotional responses to the images. When participants' responses indicated they followed a superficial ER strategy (e.g., I looked away and thought of something else), we asked them to consider strategies that would ensure they engaged with the images. Most participant responses indicated full compliance with the task instructions with appropriate strategies employed (e.g., thinking of the characters in the images as actors, or providing an alternative than the obvious scenarios per image). At the end of the training session, participants were given the opportunity to ask questions about the task.

Within 5-7 days following the initial screening, they completed a combined tDCS-fMRI session during which they performed two ERT runs for $20 \mathrm{~min}$ before and two ERT runs for 20 min during either active or sham tDCS. During the second visit, they were reminded of the task instructions and they were escorted to the MRI room where they were shown the MRI-compatible response box and practiced responding to the rating scale using the buttons on the response box.

Each trial started with a screen that instructed the subject to either "Decrease" or "Look" at the picture that followed, which was presented for $3 \mathrm{~s}$. Instructions were immediately followed by the experimental stimulus that appeared on the screen for $6 \mathrm{~s}$. Subsequently, a 4-point rating scale was presented for $3 \mathrm{~s}$, during which subjects indicated the intensity of their emotion to the picture by selecting the corresponding button on the four-button response box.

Experimental trials were interspersed with "Null" events that lasted $3 \mathrm{~s}$, in which E-prime was synchronized with the scanner. We used Optseq2 (Greve, 2002) to calculate the optimal presentation schedule in order to maximize the efficiency per acquisition in our event-related fMRI design. Out of the 100 timing files generated by Optseq2, we kept the top four. Subjects were randomly assigned to one of these four presentation schedules.

\section{tDCS parameters}

TDCS was administered in a single-blind design using a DCStimulator with an MR-compatible module (NeuroConn, $\mathrm{GmbH}$, http://www.neuroconn.de/dc-stimulator_en/). The stimulation site was determined with a BraiNet 10/20 Placement cap (https://bio-medical.com/) and was marked on the participant's scalp. For stimulation of the left DLPFC, the anode was placed over area F3 and the cathode over the contralateral mastoid (see Fig. 2 for electrical field model).
None of the participants received tDCS stimulation during the first two fMRI runs of the ER task. During the last two fMRI runs, for the active tDCS condition, stimulation was administered at $1.5 \mathrm{~mA}$ for a maximum of $20 \mathrm{~min}$ (including $10 \mathrm{~s}$ ramp-up and $10 \mathrm{~s}$ ramp-down time) via two conducting 5 $\times 5 \mathrm{~cm}$ electrodes covered with $1-\mathrm{mm}$ thick electroconductive paste (current density $=0.06 \mathrm{~mA} / \mathrm{cm}^{2}$ ). Stimulation began for $180 \mathrm{~s}$ prior to the onset of the third run of the ERT. For the sham condition, tDCS began $180 \mathrm{~s}$ prior to the onset of the third run of the ERT under the same parameters with the active condition and then, unbeknownst to the participants, was turned off.

\section{fMRI data acquisition}

Functional images were acquired on a Siemens SKYRA 3.0 T MRI system (Siemens, Erlangen, Germany) at the Hoglund Brain Imaging Center, which was equipped with a 12-channel head coil. BOLD-sensitive functional images were acquired using a single-shot gradient EPI sequence (echo time $=25 \mathrm{~ms}$; repetition time $=3 \mathrm{~s}$ ), 50 axial slices in ascending interleaved order, slice thickness $=3 \mathrm{~mm}$, field of view $=232 \mathrm{~mm}$, flip angle $=90^{\circ}$, voxel size $=2.90 \times 2.90 \times 3.00 \mathrm{~mm}^{3}$ ). Highresolution anatomical images were acquired using a MPRAGE sequence (echo time $=2.01 \mathrm{~ms}$, voxel size $=1.2$ $\times 1.0 \times 1.0 \mathrm{~mm}^{3}, 176$ sagittal slices, field of view $=256 \mathrm{~mm}$ ).

\section{fMRI data analysis}

Functional data were preprocessed and analyzed with the AFNI software package (Cox, 1996). A standardized preprocessing pipeline involved de-spiking of the data by fitting a smooth-ish curve to each voxel time series, alignment of slice timings of volumes, masking for removal of the skull, and registration of functional images to the anatomy (Saad et al., 2009). Subsequently, functional images were co-registered (Cox \& Jesmanowicz, 1999) and projected into standard stereotaxic space (Talairach \& Tournoux, 1988). The normalized images were smoothed with an isotropic 5-mm FWHM Gaussian kernel, and converted to percent signal change. The ensuing preprocessed fMRI time series were analyzed on a subject-by-subject basis using an event-related approach in the context of voxel-wise multiple linear regression with regressors for each condition (positive look, positive decrease, neutral look, negative look, and negative decrease) convolved with a canonical hemodynamic response function. In addition, we had regressors for the instructions and null events. Six motion parameters and their derivatives and the signal extracted from an eroded CSF mask were included as noise covariates of no interest. We used the program 3dMEMA (allowing for the modeling of both within- and across-subjects variability) to conduct general linear tests to obtain the main effect of ER on positive (positive decrease - positive look) and negative pictures (negative decrease - negative look) and their 


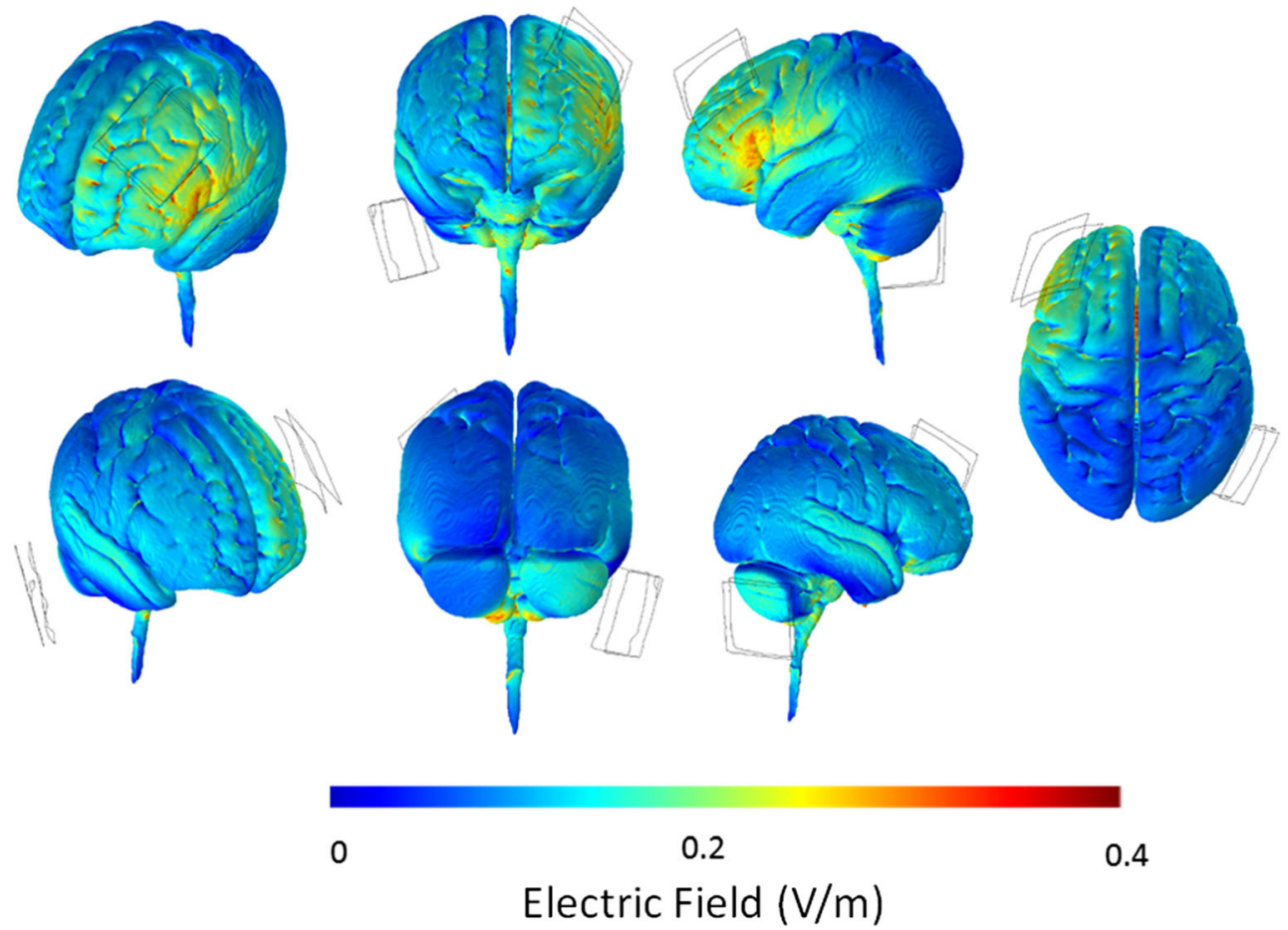

Fig. 2 Electrical field model induced by tDCS, showing points of high current density (peak current flow) under the unilateral montage used in the present study at $1.5 \mathrm{~mA}$ with anodal tDCS over F3 in the left hemisphere, and the reference electrode over the contralateral (right)

interaction with patient status (MDD patients vs. healthy controls).

In a random-effects analysis, group maps were created by comparing activations against a constant value of 0 . The group maps were thresholded at voxelwise $p<0.02$ and corrected for multiple comparisons by removing clusters smaller than 1,538 $\mu \mathrm{L}$ to achieve a mapwise corrected two-tailed $p<0.05$. We used AFNI program 3dFWHMx to calculate the autocorrelation function (ACF) parameters for each individual subject. Subsequently, the median ACF value of all subjects was used in running the $3 \mathrm{dClustSim}$ program with 10,000 iterations (see Cox, Reynolds, \& Taylor, 2016). The cluster threshold was determined through Monte Carlo simulations that estimate the chance probability of spatially contiguous voxels exceeding the voxelwise $p$ threshold. The analysis was restricted to a mask that excluded areas outside the brain, as well as deep white matter areas and the ventricles. This mask is based on the probabilistic Desikan-Killiany atlas that contains 35 cortical areas in each hemisphere (Desikan et al., 2006), and the subcortical parcellation provided by FreeSurfer. The mask was created using TT desai dkpmaps atlas provided with AFNI.

\section{Region-of-interest (ROI) analysis}

To investigate any potential differences in the recruitment of emotional processing regions between patients and mastoid. The plots reflect the electric norm or the absolute magnitude of electric field. This custom model was created with HD-Explore software version 5 (Soterix Medical, https://soterixmedical.com/research/ software/hd-explore/releasenotes). $\mathrm{V} / \mathrm{m}=$ volts/meter

control subjects across conditions, we defined a left and right ROI within the amygdala using the probabilistic Desikan-Killiany atlas (Desikan et al., 2006) as provided in AFNI. For healthy controls we hypothesized stronger amygdala activity when looking at negative pictures compared to decreasing emotional responses to the pictures, whereas no such difference (or a reversed pattern of results) was expected for MDD patients. Given the inconsistencies in the literature regarding the role of the amygdala in processing positive emotions, we didn't have any clear hypotheses with regard to the direction of the main effect of ER and the regulation $\times$ group interaction effect for positive stimuli. Parameter estimates for the negative decrease, negative look, positive decrease, positive look, and neutral look conditions were extracted separately from all voxels in the left and right amygdala ROI. Given our a priori prediction of greater amygdala activation in negative-look compared to negative-decrease trials for healthy controls, we entered mean parameter estimates for the decrease and look conditions within the ROI as dependent variables in a one-tailed paired-sample $t$-test separately for MDD patients and controls. Mean parameter estimates for the positive-decrease and positive-look conditions with the ROI were entered as dependent variables in a two-tailed paired-sample $t$-test separately for MDD patients and controls. We used a false discovery rate (FDR) correction for multiple comparisons (Benjamini \& 
Hochberg, 1995), with FDR of 5\% and corrected critical $p$ value of .01 .

\section{Functional connectivity analysis}

To investigate which brain areas show a modulation of the functional connectivity to the bilateral amygdala and the left DLPFC by Emotion Regulation and are affected by active tDCS, we conducted a generalized psychophysiological interaction (gPPI) analysis (Friston, 1994; McLaren et al., 2012; O'Reilly et al., 2012). For our first physiological seed we extracted the de-trended and deconvolved time series for each subject from a ROI, based on a bilateral amygdala mask created using the probabilistic Desikan-Killiany atlas (Desikan et al., 2006) as provided in AFNI. For our second physiological seed we extracted the de-trended and de-convolved time series for each subject from an ROI, based on a 6-mm sphere around the peak activations observed within the left DLPFC for the negative decrease - negative look contrast from our univariate analysis during the pre-stimulation runs across all subjects (left DLPFC $(-36,13,45)$ ). We calculated the psychophysiological interaction term for each condition by multiplying the condition regressor (psychological variable) with the time course in our seed region (physiological variable). For both of our seed regions separately, we added the newly created regressors that capture the psychophysiological interaction terms and the physiological regressor (i.e., seed time course) to the model that included all the original regressors. We were interested in whether functional connectivity to the bilateral amygdala and left DLPFC is modulated by ER (i.e., differential connectivity during decrease as compared to look trials), and whether this psychophysiological interaction (PPI) is different for controls versus MDD patients. We used the program 3dMEMA to conduct a group analysis with a mixed-effect design with one fixed factor (group: MDD patients vs. controls) and one random factor (subject). This analysis allowed us to identify brain areas that showed a differential change in functional connectivity to the bilateral amygdala or left DLPFC as a function of the experimental context (decrease vs. look trials) between MDD patients and healthy controls. Separate analyses were conducted for positive and negative emotional stimuli. To investigate the effect of stimulation on functional connectivity to the bilateral amygdala and left DLPFC, we entered the parameter estimates associated with the psycho-physiological interaction terms of the stimulation during reappraisal (i.e., negative decrease - negative look during stimulation vs. negative decrease - negative look pre-stimulation) and the stimulation during positive reappraisal contrast (i.e., positive decrease - positive look during stimulation vs. positive decrease - positive look pre-stimulation) into a separate whole-brain-group analysis. In this group analysis we used a mixed-effect model with two fixed factors (stimulation: subjects who received active stimulation vs. subjects who received sham stimulation; group: MDD patients vs. healthy controls) and one random factor (subject). First, this analysis allowed us to identify brain areas that showed a differential change in connectivity to the bilateral amygdala or left DLPFC as a function of the experimental context (stimulation during ER vs. no stimulation during ER) between subjects who received active versus subjects who received sham stimulation. Second, the Emotion regulation $\times$ tDCS Stimulation $\times$ Group interaction allowed us to investigate a potential differential effect of tDCS stimulation on modulating ER networks between MDD patients and healthy controls.

\section{Results}

\section{Behavioral results}

\section{Pre-stimulation trials}

Affect ratings for healthy control participants by trial type A paired two-tailed Student's $t$-test on the mean affect ratings of healthy controls indicated that negative pictures elicited less negative affect in decrease trials $(M=2.28, S D=0.50)$ compared to look trials $(M=2.91, S D=0.45, t(17)=5.19, p<$ 0.001 , Cohen's $d=1.32$ ), and positive pictures elicited less positive affect in decrease trials $(M=1.89, S D=0.45)$ compared to look trials $(M=2.47, S D=0.57, t(17)=4.88, p<$ 0.001, Cohen's $d=1.13$; see Fig. 3). Furthermore, neutral pictures elicited less negative affect $(M=1.60, S D=0.43)$ compared to negative decrease $(t(17)=3.96, p<0.005$, Cohen's $d=1.43)$ and negative-look trials $(t(17)=9.24, p<$ 0.001 , Cohen's $d=2.72$ ). Neutral pictures elicited less positive affect $(M=1.60, S D=0.43)$ compared to positive decrease $(t(17)=2.33, p<0.05$, Cohen's $d=0.64)$ and positivelook trials $(t(17)=8.23, p<0.001$, Cohen's $d=1.69)$.

Affect ratings for MDD patients by trial type A paired twotailed Student's $t$-test on the mean affect ratings of MDD patients indicated that negative pictures elicited less negative affect in decrease trials $(M=2.31, S D=0.41)$ compared to look trials $(M=2.94, S D=0.56, t(18)=7.78, p$ $<0.001$, Cohen's $d=1.28$; see Fig. 3 ), and positive pictures elicited less positive affect in decrease trials $(M=$ $1.91, S D=0.50)$ compared to look trials $(M=2.42, S D=$ $0.71, t(18)=4.79, p<0.001$, Cohen's $d=0.83$; see Fig. $3)$. Furthermore, neutral pictures elicited less negative affect $(M=1.53, S D=0.38)$ compared to negative decrease $(t(17)=7.94, p<0.001$, Cohen's $d=1.97)$ and negative- 


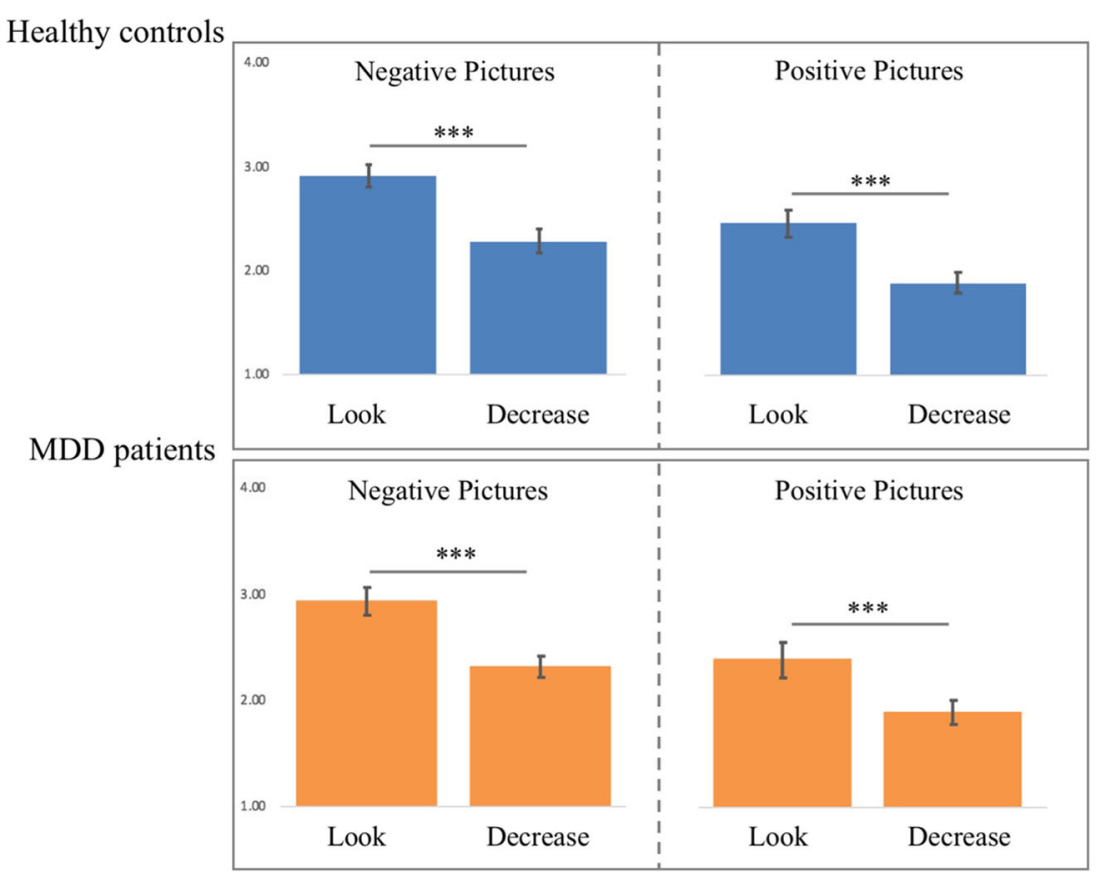

Fig. 3 Mean affect ratings of healthy controls and major depressive disorder (MDD) patients to positive and negative Look and Decrease trials (error bars depict the standard error of the mean) during the pre-stimulation trials. * indicates $\mathrm{p}<.05, * *$ indicates $\mathrm{p}<.01, * * *$ indicates $\mathrm{p}<.001$

look trials $(t(17)=10.90, p<0.0001$, Cohen's $d=2.95)$. Neutral pictures elicited less positive affect $(M=1.53, S D$ $=0.38)$ compared to positive decrease $(t(17)=4.04, p<$ 0.001 , Cohen's $d=0.86)$ and positive-look trials $(t(17)=$ $5.86, p<0.001$, Cohen's $d=1.56$ ).

Reappraisal scores by participant group Subsequently, we calculated each subject's reappraisal score (for negative and positive pictures) by subtracting the mean rating obtained in decrease trials from the mean rating obtained in look trials. An independent-sample two-tailed Student's $t$-test on the reappraisal scores showed that healthy controls $(M=0.63, S D=$ $0.51)$ and depressed patients $(M=0.62, S D=0.35)$ did not differ in their negative reappraisal scores $(t(18)=0.02, p>.90$, Cohen's $d=0.02)$. Similarly, healthy controls $(M=0.58, \mathrm{SD}=$ $0.50)$ and depressed patients $(M=0.50, S D=0.46)$ did not differ in their positive reappraisal scores $(t(18)=0.47, p>$ 0.60 , Cohen's $d=0.17$ ). Overall, across trial types, affect and reappraisal scores did not differ between healthy control participants and MDD patients (see Fig. 3).

\section{During stimulation trials}

Affect ratings for healthy control participants by trial type A paired-samples two-tailed $t$-test on the mean affect ratings of healthy controls made during the stimulation runs indicated that negative pictures elicited less negative affect in the decrease trials $(M=2.24, S D=0.50)$ than in the look trials $(M=$ $2.99, S D=0.51, t(17)=5.96, p<.0001$, Cohen's $d=1.49$; see Fig. 4). Similarly, positive pictures elicited less positive affect in the decrease trials $(M=1.84, S D=0.49$; see Fig. 4$)$ than in the look trials $(M=2.45, S D=0.62, t(17)=5.75, p<.0001$, Cohen's $d=1.09)$. Furthermore, neutral pictures elicited less negative affect $(M=1.64, S D=0.45)$ compared to negative decrease $(t(17)=3.68, p<0.005$, Cohen's $d=1.26)$ and negative-look trials $(t(17)=11.08, p<0.0001$, Cohen's $d=$ $2.81)$. Neutral pictures elicited less positive affect $(M=1.64$, $S D=0.45)$ compared to positive-look trials $(t(17)=8.57, p<$ 0.001 , Cohen's $d=1.50$ ).

Affect ratings for MDD patients by trial type A paired-samples two-tailed $t$-test on the mean affect ratings of MDD patients made during the stimulation runs indicated that negative pictures elicited less negative affect in the decrease trials $(M=$ $2.22, S D=0.51)$ than in the look trials $(M=2.84, S D=0.68$, $t(18)=6.20, p<.00001$, Cohen's $d=1.03$; see Fig. 4). Similarly, positive pictures elicited less positive affect in the decrease trials $(M=1.70, S D=0.57)$ than in the look trials $(M$ $=2.21, S D=0.64, t(18)=5.46, p<.0001$, Cohen's $d=0.84$; see Fig. 4). Furthermore, neutral pictures elicited less negative affect $(M=1.51, S D=0.36)$ compared to negative decrease $(t(17)=6.64, p<0.001$, Cohen's $d=1.61)$ and negative-look trials $(t(17)=9.08, p<0.0001$, Cohen's $d=2.44)$. Similarly, neutral pictures elicited less positive affect $(M=1.51, S D=$ $0.36)$ compared to positive decrease $(t(17)=2.34, p<0.05$, Cohen's $d=0.40)$ and positive-look trials $(t(17)=6.49, p<$ 0.001 , Cohen's $d=1.35$ ).

Reappraisal scores by participant group An independentsample two-tailed $t$-test on the reappraisal scores showed that 
HEALTHY CONTROLS

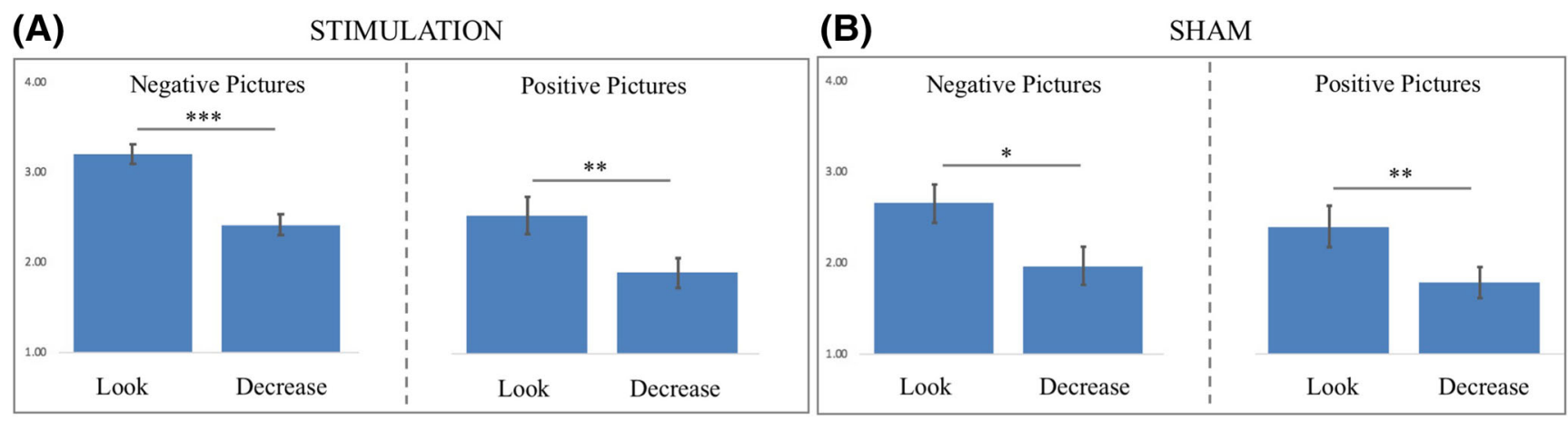

MDD PATIENTS

(C) STIMULATION (D)
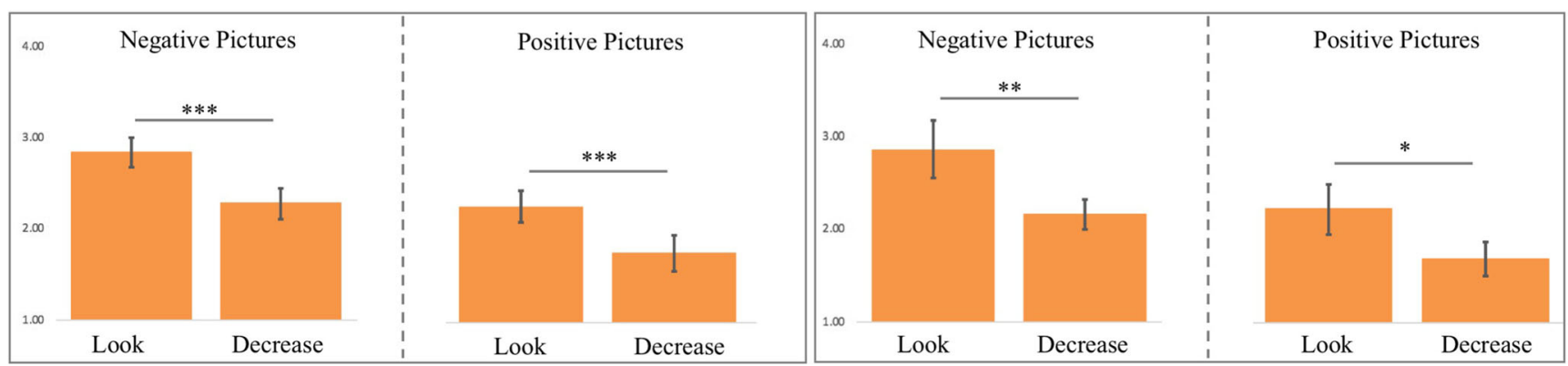

Fig. 4 Mean affect ratings for the negative look, negative decrease, positive look, and positive-decrease trials during stimulation for (A) healthy controls who received active stimulation, (B) healthy controls who received sham stimulation, (C) major depressive disorder (MDD)

patients who received active stimulation, and (D) MDD patients who received sham stimulation (error bars depict the standard error of the mean). * indicates $p<.05, * *$ indicates $p<.01, * * *$ indicates $p<.001$

healthy controls $(M=0.75, S D=0.53)$ and MDD patients $(M$ $=0.63, S D=0.44)$ did not differ in their negative reappraisal scores $(t(19)=0.76, p=.23$, Cohen's $d=0.25)$ during tDCS. Similarly, healthy controls $(M=0.61, S D=0.45)$ and MDD patients $(M=0.51, S D=0.41)$ did not differ in their positive reappraisal scores $(t(19)=0.72, p=.24$, Cohen's $d=0.23$ ) during tDCS.

\section{Effect of stimulation on ER (ER) performance}

To investigate any direct effect of stimulation on ER performance we conducted a repeated-measures analysis of variance (ANOVA) on the reappraisal scores (decrease-look) with one within-subjects factor (time: pre-stimulation vs. during stimulation) and two between-subjects factors (group: patients vs. controls; stimulation: active vs. sham stimulation). The interactions between group and time $(F[1,33]=1.06, M S E=$ $\left.0.058, \eta_{p}^{2}=.033, p>.30\right)$ and stimulation and time $(F[1,33]$ $\left.=0.09, M S E=0.007, \eta_{p}^{2}=.004, p>.70\right)$ in a repeatedmeasures ANOVA of the negative reappraisal scores were not significant. Similarly, the three-way group $\times$ stimulation $\times$ time interaction was not significant, $F(1,33)=0.036, M S E=$ $0.002, \eta_{p}^{2}=.001, p>.80$.

\section{fMRI results}

\section{Main effect of ER prior to stimulation}

To elucidate the neural network involved in ER of positive and negative events, we contrasted the Decrease and Look conditions across all subjects (i.e., negative decrease - negative look and positive decrease - positive look). Caret (Van Essen et al., 2001) was used to display the activation maps of our various contrasts (including the PPI analyses) on an inflated cortical surface created through FreeSurfer (Fischl, 2012).

Negative decrease $>$ negative look Stronger activation for negative-decrease trials versus negative-look trials was observed in the bilateral inferior parietal lobule (IPL), angular gyrus (AnG), middle frontal gyrus (MFG), left superior frontal gyrus (SFG), inferior frontal gyrus (pars triangularis), middle temporal gyrus (MTG), as well as the right insula (Fig. 5A; Table 1).

Negative look $>$ negative decrease Stronger activation for negative-look trials versus negative-decrease trials was observed in the left calcarine gyrus and postcentral gyrus (Fig. $5 \mathrm{~A}$; Table 1). 
Table 1. The volume of the cluster $(\mu \mathrm{l})$, peak z-score, Talairach coordinates, and the anatomical structures that the clusters overlap are shown. $\mathrm{L}=$ left hemisphere, $\mathrm{R}=$ right hemisphere

\begin{tabular}{|c|c|c|c|c|c|}
\hline Volume & Max & $\mathrm{x}$ & $\mathrm{y}$ & $\mathrm{z}$ & Structure \\
\hline \multicolumn{6}{|c|}{ Negative Decrease > Negative Look } \\
\hline 35172 & 4.74 & -6 & 28 & 41 & L superior frontal gyrus \\
\hline 9141 & 5.44 & 51 & -51 & 38 & $\mathrm{R}$ inferior parietal lobule; angular gyrus \\
\hline 6859 & 4.95 & -48 & -51 & 43 & L inferior parietal lobule; angular gyrus \\
\hline 4844 & 4.35 & -28 & 53 & 3 & $\mathrm{~L}$ middle frontal gyrus \\
\hline 2750 & 3.96 & -46 & 23 & 3 & L inferior frontal gyrus (pars triangularis) \\
\hline 2141 & 3.73 & 36 & 48 & 1 & $\mathrm{R}$ middle frontal gyrus \\
\hline 1875 & 4.10 & 48 & 8 & 3 & R Insula \\
\hline 1719 & 4.00 & -58 & -51 & 3 & L middle temporal gyrus \\
\hline \multicolumn{6}{|c|}{ Negative Look $>$ Negative Decrease } \\
\hline 26141 & 5.20 & -8 & -53 & 3 & L Calcarine gyrus \\
\hline 3688 & 4.22 & -53 & -16 & 11 & L postcentral gyrus \\
\hline \multicolumn{6}{|c|}{ Positive Decrease $>$ Positive Look } \\
\hline 16906 & 4.55 & 43 & 16 & 43 & L middle frontal gyrus \\
\hline 11469 & 6.15 & 56 & -53 & 31 & $\mathrm{R}$ inferior parietal lobule; angular gyrus \\
\hline 10219 & 5.81 & -41 & -53 & 26 & L inferior parietal lobule; angular gyrus \\
\hline 5578 & 4.65 & 43 & 41 & -1 & $\mathrm{R}$ middle frontal gyrus \\
\hline 4188 & 5.29 & -61 & -31 & 1 & $\mathrm{~L}$ middle temporal gyrus \\
\hline 3656 & 4.74 & -33 & 56 & 1 & L middle frontal gyrus \\
\hline 3219 & 4.03 & 63 & -23 & -8 & $\mathrm{R}$ middle temporal gyrus \\
\hline 3109 & 3.85 & 18 & 43 & 33 & $\mathrm{R}$ superior frontal gyrus \\
\hline 2844 & 5.33 & -36 & -58 & -41 & L Cerebellum \\
\hline 2766 & 4.50 & -41 & 21 & 48 & L middle frontal gyrus \\
\hline 2672 & 5.13 & -51 & 16 & -13 & L superior temporal gyrus \\
\hline \multicolumn{6}{|c|}{ Positive Look > Positive Decrease } \\
\hline 35078 & 5.67 & 8 & -61 & 13 & $\mathrm{R}$ precuneus \\
\hline 3594 & 4.84 & -36 & -16 & 18 & L Insula \\
\hline 3125 & 4.54 & 38 & -61 & -1 & $\mathrm{R}$ inferior temporal gyrus \\
\hline \multicolumn{6}{|c|}{ Regulation $\times$ group interaction (negative pictures) } \\
\hline 13516 & 4.75 & 16 & -81 & -1 & $\mathrm{R}$ lateral occipital cortex \\
\hline
\end{tabular}

Positive decrease $>$ positive look Stronger activation for positive-decrease trials versus positive-look trials was observed in the bilateral IPL, AnG, MFG, MTG, left superior temporal gyrus (STG), cerebellum, as well as the right SFG (Fig. 5B; Table 1).

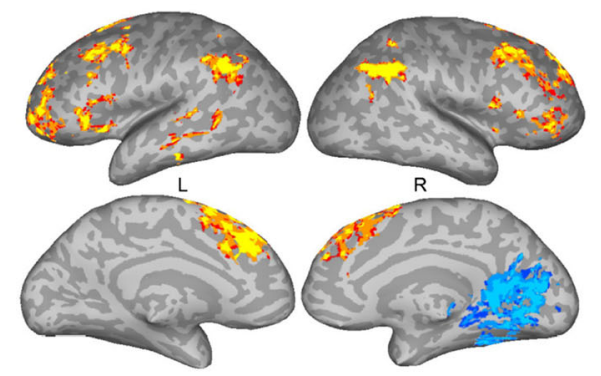

(A) Negative Decrease - Negative Look

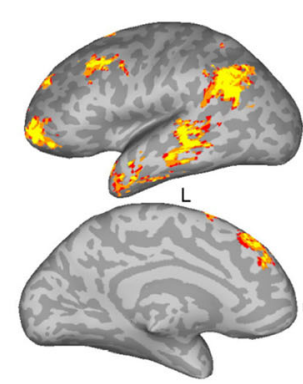

(B) Positive Decrease - Positive Look

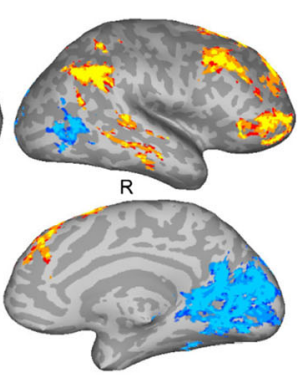

$P<0.02 \quad P<0.01 \quad P<0.001$

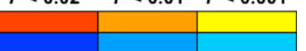

Fig. 5 Activations for (A) Negative Decrease-Negative Look (B) Positive Decrease - Positive Look trials prior to stimulation across groups. Redorange colors denote stronger activation for the Decrease condition, blue- cyan colors denote stronger activation for the Look condition. Activations are projected on an inflated surface of a brain. Gyri are shown in light gray and sulci in dark gray. $\mathrm{L}=$ left hemisphere, $\mathrm{R}=$ right hemisphere 

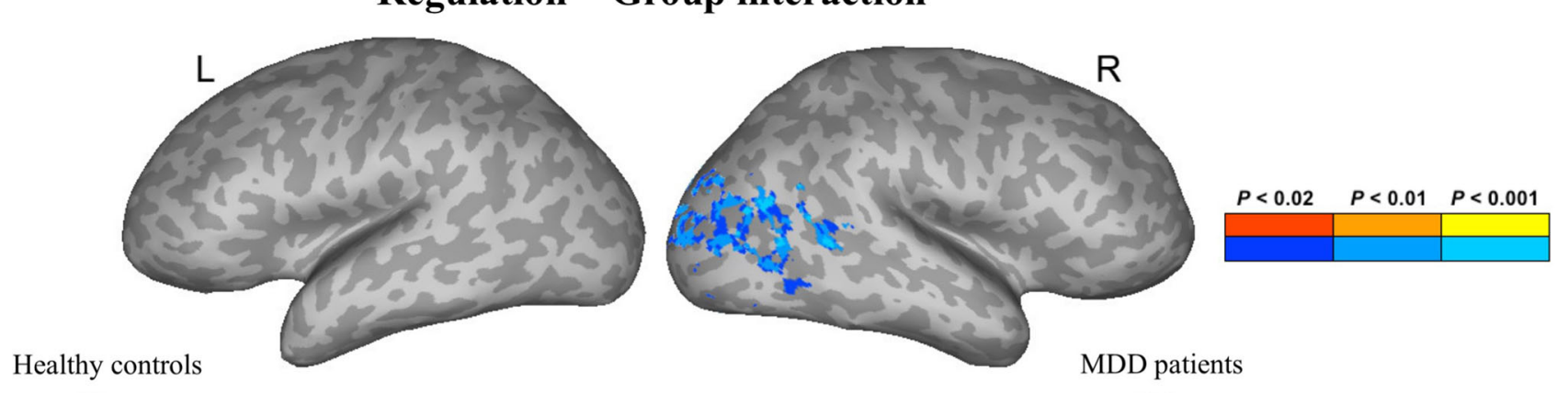

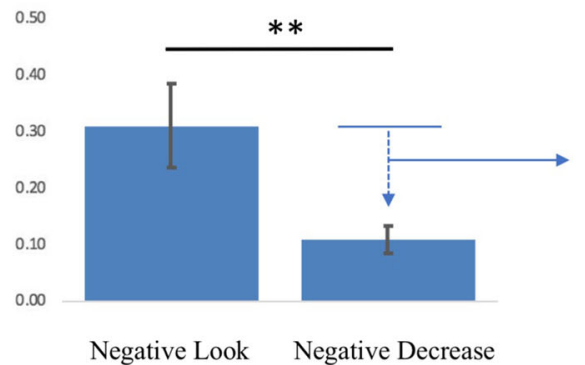

Fig. 6 Activations for the emotion regulation $(E R) \times$ group interaction for negative pictures (i.e., negative decrease - negative look (healthy controls) - negative decrease - negative look (major depressive disorder (MDD) patients). Activations are projected on an inflated surface of a brain. Gyri are shown in light gray and sulci in dark gray. Parameter
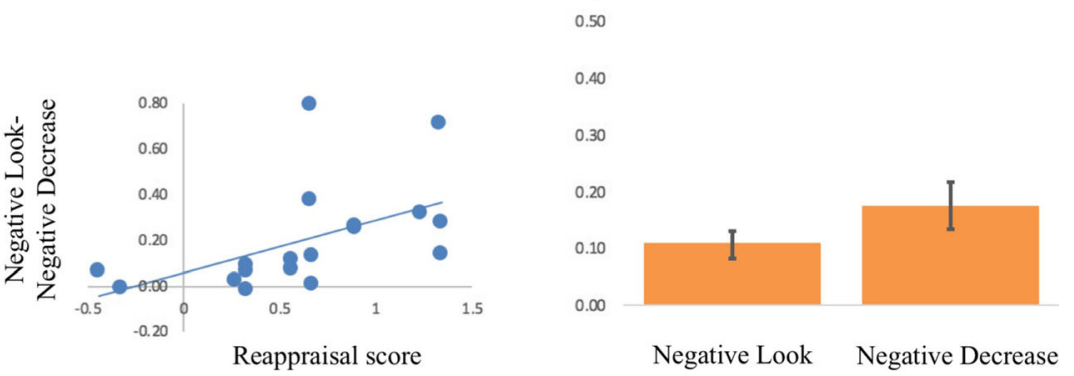

estimates for the negative-look and negative-decrease conditions for healthy controls and MDD patients averaged over voxels that showed an ER $\times$ group interaction effect. The amount of down-regulation of activity in the visual cortex of healthy controls was positively correlated with their reappraisal score $(r=0.52, p=0.02)$. ** denotes $p<.01$

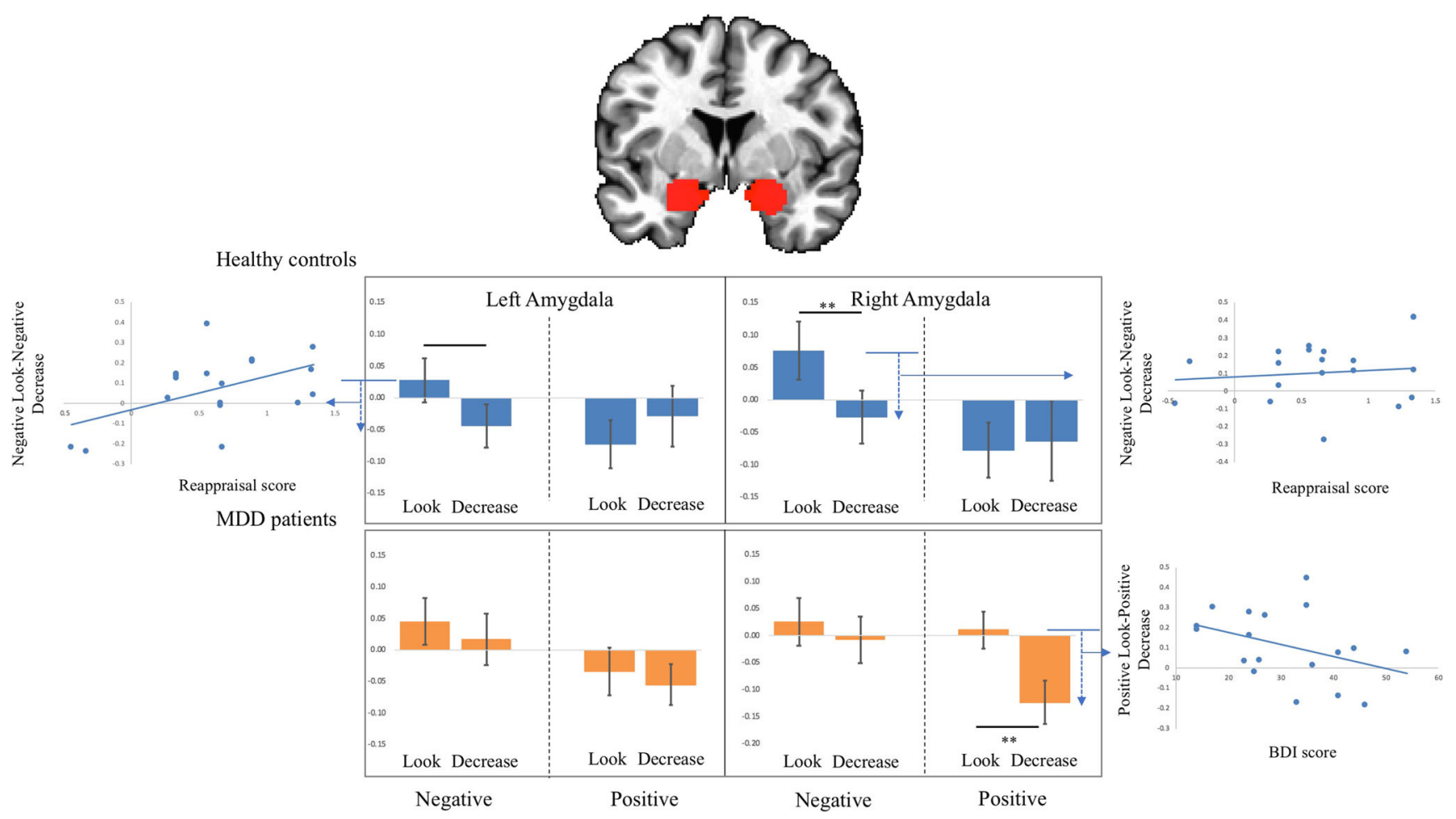

Fig. 7 Results of the region-of-interest (ROI) analysis for the prestimulation trials within a left and right amygdala mask. Parameter estimates for the look and decrease conditions are plotted separately for the negative and positive pictures and for the healthy controls and major depressive disorder (MDD) patients. Healthy controls showed a significant down-regulation effect of negative pictures within the right amygdala, $t(17)=2.69, p=0.008$; the effect did not reach statistical significance for the left amygdala, $t(17)=1.79, p=0.046$. The amount of down- regulation in the left amygdala of healthy controls during processing of negative pictures was positively correlated with the participants' reappraisal score $(r=0.50, p=0.035)$. The amount of down-regulation in the right amygdala of MDD patients during processing of positive pictures was not significantly correlated with their BDI score $(r=-0.39, p=$ $0.11) * p<.01 ; * * p<.001$, FDR-corrected for multiple comparisons at $\mathrm{FDR}=.05$ with a corrected critical $p$ value of .01 (Benjamini-Hochberg, 1995) 
Table 2 The volume of the cluster $(\mu \mathrm{l})$, peak $\mathrm{z}$-score, Talairach coordinates, and the anatomical structures that the clusters overlap are shown. $\mathrm{L}=$ left hemisphere, $\mathrm{R}=$ right hemisphere

\begin{tabular}{|c|c|c|c|c|c|}
\hline Volume & Max & $\mathrm{x}$ & $\mathrm{y}$ & $\mathrm{z}$ & Structure \\
\hline \multicolumn{6}{|c|}{ Negative Decrease > Negative Look (controls) } \\
\hline 5656 & 4.49 & -3 & 18 & 38 & $\mathrm{~L}$ anterior cingulate cortex \\
\hline 5094 & 3.77 & 48 & -61 & 31 & $\mathrm{R}$ inferior parietal lobule; angular gyrus \\
\hline 2141 & 4.11 & 48 & 23 & 41 & R superior frontal gyrus \\
\hline 1641 & 4.34 & -48 & 18 & 36 & L superior frontal gyrus \\
\hline \multicolumn{6}{|c|}{ Negative Look > Negative Decrease (controls) } \\
\hline 48812 & 5.19 & 18 & -68 & 11 & R lateral occipital cortex \\
\hline 2359 & 5.20 & -61 & -8 & 26 & L middle frontal gyrus \\
\hline 1688 & 4.55 & -36 & -58 & -11 & L lateral occipital cortex \\
\hline \multicolumn{6}{|c|}{ Positive Decrease > Positive Look (controls) } \\
\hline 11250 & 4.15 & 48 & 16 & 38 & $\mathrm{R}$ middle frontal gyrus \\
\hline 8562 & 4.93 & 51 & -53 & 38 & $\mathrm{R}$ inferior parietal lobule; angular gyrus \\
\hline 6516 & 4.95 & -56 & -53 & 41 & $\mathrm{~L}$ inferior parietal lobule; angular gyrus \\
\hline 4016 & 4.29 & 43 & 41 & 3 & R inferior frontal gyrus (pars orbitalis) \\
\hline 3141 & 4.14 & 66 & -36 & 1 & $\mathrm{R}$ middle temporal gyrus \\
\hline 2250 & 4.28 & -53 & -28 & -6 & L superior temporal sulcus \\
\hline 2000 & 4.40 & -46 & -1 & -23 & L middle temporal gyrus \\
\hline 1594 & 4.06 & -36 & 56 & 8 & L middle frontal gyrus \\
\hline \multicolumn{6}{|c|}{ Positive Look > Positive Decrease (controls) } \\
\hline 25203 & 4.83 & -13 & -86 & -11 & L lateral occipital cortex \\
\hline 2203 & 4.24 & 51 & -58 & 1 & $\mathrm{R}$ middle temporal gyrus \\
\hline 1812 & 3.89 & -41 & -16 & 18 & L postcentral gyrus \\
\hline \multicolumn{6}{|c|}{ Negative Decrease > Negative Look (patients) } \\
\hline 5328 & 5.03 & 48 & -53 & 31 & $\mathrm{R}$ inferior parietal lobule; angular gyrus \\
\hline 3547 & 4.44 & -6 & 31 & 41 & $\mathrm{~L}$ anterior cingulate cortex \\
\hline 2797 & 4.04 & 28 & 41 & 33 & $\mathrm{R}$ middle frontal gyrus \\
\hline 2625 & 4.36 & -48 & -51 & 43 & $\mathrm{~L}$ inferior parietal lobule; angular gyrus \\
\hline 2375 & 4.19 & -28 & 53 & 3 & L middle frontal gyrus \\
\hline 1594 & 3.82 & 18 & 8 & 66 & $\mathrm{R}$ superior frontal gyrus \\
\hline \multicolumn{6}{|c|}{ Positive Decrease > Positive Look (patients) } \\
\hline 3234 & 4.53 & 56 & -53 & 31 & $\mathrm{R}$ inferior parietal lobule; angular gyrus \\
\hline 2750 & 3.70 & 21 & 46 & 23 & R superior frontal gyrus \\
\hline 2266 & 4.52 & -41 & -53 & 26 & $\mathrm{~L}$ inferior parietal lobule; angular gyrus \\
\hline 1625 & 4.55 & -58 & -31 & 8 & L superior temporal gyrus \\
\hline \multicolumn{6}{|c|}{ Positive Look > Positive Decrease (patients) } \\
\hline 7797 & 4.34 & 11 & -66 & 16 & $\mathrm{R}$ lateral occipital cortex \\
\hline
\end{tabular}

Positive look $>$ positive decrease Stronger activation for positive-look trials versus positive-decrease trials was observed in the left insula, as well as the right precuneus and inferior temporal gyrus (ITG) (Fig. 5B; Table 1).

Lastly, to examine whether primary visual cortex activation indeed reflected a strengthened visual response to pictures that are relatively high in emotional intensity (with either positive or negative valence) across both participant groups, we ran additional analyses in which we contrasted the Negative or Positive Look conditions with the Neutral Look condition across and within groups. These analyses showed that merely looking at negative and positive pictures elicited a differential response within primary visual areas compared to looking at neutral pictures for both healthy participants and MDD patients (see Supplementary Figs. 3-5, Online Supplementary Material), which confirms that visual cortex activity tracks the emotional intensity of the stimuli for both groups. 


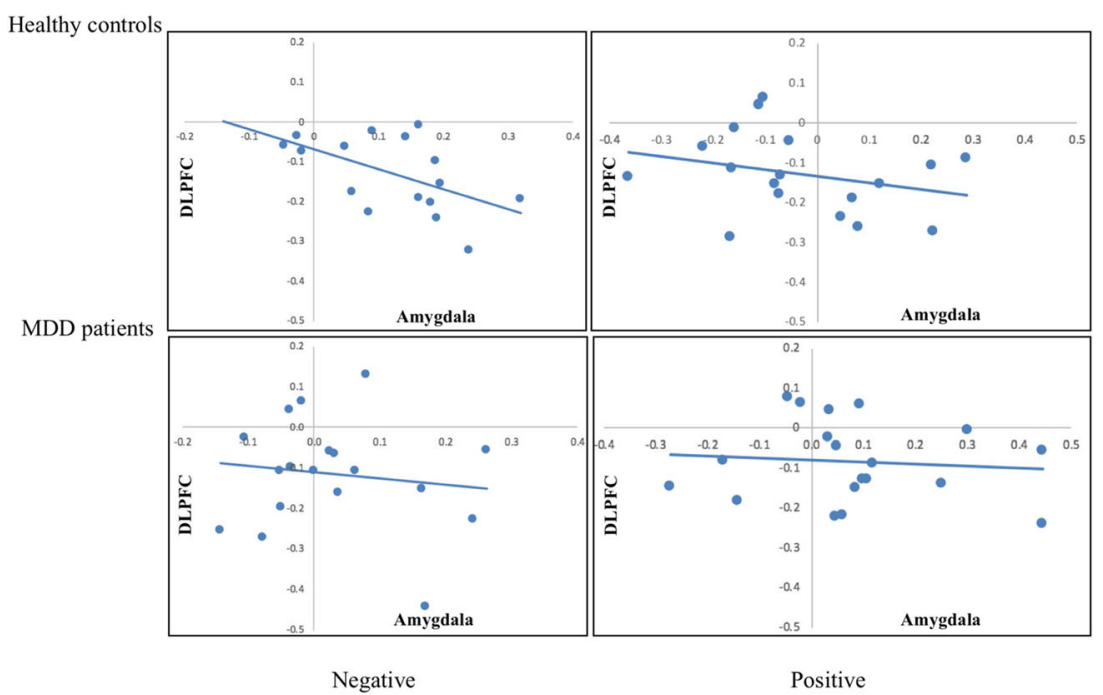

Fig. 8 Prior to stimulation, healthy controls showed a significant relationship between the degree of down-regulation to negative pictures within the bilateral amygdala and the degree of up-regulation in the bilateral dorsolateral prefrontal cortex (DLPFC), $r=-0.54, t(14)=2.43, p=0.03$

\section{$E R \times$ group interaction prior to stimulation}

To investigate any potential differences in the strength or extent of the neural network involved in ER between patients and control participants during the pre-stimulation runs, we ran a mixed-effect group analysis on the Decrease - Look contrast with one fixed factor (group: patients vs. controls) and one random factor (subject).

The ER $\times$ group interaction for negative pictures (i.e., negative decrease - negative look (healthy controls) vs. negative decrease - negative look (MDD patients)) yielded a significant effect within the lateral occipital cortex, cuneus, lingual gyrus, and the primary visual cortex (Figs. 6 and 7; Table 1). Healthy control subjects demonstrated a reduction of activity within the visual cortex as a function of ER (i.e., higher parameter estimate for the negative look $(M=0.31)$ as compared to the negative decrease $(M=0.11)$ condition, $t(17)=3.70, p=$ .002 , Cohen's $d=0.52$ ), whereas MDD patients demonstrated the opposite pattern of results (i.e., lower parameter estimate for the negative look $(M=0.11)$ as compared to the negative decrease $(M=0.18)$ condition, $t(18)=2.42, p=.027$, Cohen's $d=0.25$ ). Subsequently, we were interested in examining whether this reduction of activity within the visual cortex as a function of ER was correlated with participants' ER scores. For healthy control participants, the Pearson's productmoment correlation between the average amount of downregulation (i.e., negative look - negative decrease) over voxels that showed an ER $\times$ group interaction effect and a subjects' reappraisal score for negative pictures (i.e., rating for negative look - negative decrease) was significant, $r=0.52, t(16)=$ $2.44, p=0.027$. For MDD patients, this correlation was not significant, $r=0.17, t(17)=0.71, p=0.49$. These findings suggest that control subjects were able to effectively reduce the signal within primary visual regions during ER in response to negative stimuli, whereas MDD patients were not able to do so. What's more, the effectiveness of control participants' ability to regulate their emotion (as measured by their reappraisal score) was correlated with the degree to which the signal within primary visual areas was reduced as a function of down-regulation of the participants' emotion to negative pictures.

The ER $\times$ group interaction for positive pictures (i.e., positive decrease - positive look [controls] vs. positive decrease positive look [patients]) did not yield any areas of activation that survived our cluster-level threshold. Furthermore, we contrasted the decrease and look condition for negative and positive pictures separately for controls and patients. Table 2 provides an overview of the areas involved in ER of positive and negative pictures for controls and patients (also see Supplementary Figs. 1 and 2, Online Supplementary Material).

\section{$\mathrm{ROI}$ analysis prior to stimulation}

For this analysis we used an FDR correction for multiple comparisons of $5 \%$ and a corrected critical $p$ value of .01 (Benjamini \& Hochberg, 1995). A one-tailed paired-sample $t$-test demonstrated that healthy controls elicited stronger activation in the right amygdala to negative pictures in the look compared to the decrease condition, $t(17)=2.69, p=.008$, Cohen's $d=0.57$. In contrast, for healthy control participants, the activation within the left amygdala to negative pictures in the Look compared to the Decrease condition did not reach statistical significance following multiple comparison correction, $t(17)=1.79, p=0.046$, Cohen's $d=0.50$. The data from one healthy control subject were removed from the 

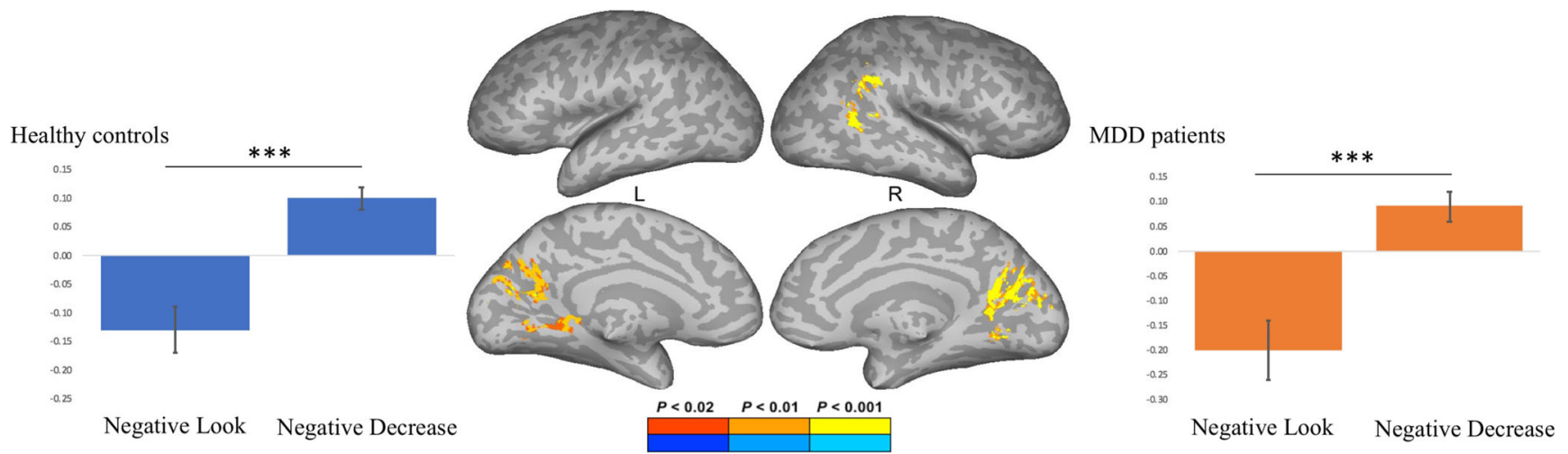

Fig. 9 Psychophysiological interaction analysis prior to stimulation showing that activity in the left dorsolateral prefrontal cortex (DLPFC) was accompanied by task-dependent functional connectivity with the cuneus, lingual gyrus, primary visual cortex, as well as the right inferior

correlational analysis of the right amygdala and the negative reappraisal score, because they deviated more than 2 standard deviations from the mean. For healthy controls, the degree to which activity in the left amygdala was down-regulated was positively correlated with the participants' reappraisal scores, $r$ $=0.50, t(16)=2.31, p=0.035$. In contrast, the degree to which activity in the right amygdala was down-regulated was not correlated with the participants' reappraisal scores, $r=0.16$, $t(15)=0.63, p=0.54$. No differences were observed in activity of either the left or right amygdala to positive pictures in the look compared to the decrease condition (all $p \mathrm{~s}>0.1$ ).

For MDD patients, we did not observe any differences in activity of either the left or right amygdala in response to negative pictures in the look compared to the decrease condition (all $p \mathrm{~s}>0.1$ ). A two-tailed paired-sample $t$-test demonstrated that MDD patients elicited stronger activation within the right amygdala to positive pictures in the look compared to the decrease condition, $t(18)=2.91, p=0.009$, Cohen's $d=$ 0.83 . No difference was observed in the left amygdala to positive pictures in the look compared to the decrease condition $(p$ $>0.3$ ). The data from one MDD patient were removed from the correlational analyses between the right amygdala and the parietal lobule (IPL) and angular gyrus. Parameter estimates of the psychophysiological interaction analysis for the negative-look and negativedecrease condition for healthy controls and major depressive disorder (MDD) patients. $* * *$ denotes $p<.001$

reappraisal/BDI score, because they deviated more than 2 standard deviations from the mean. The degree to which activity in the right amygdala was down-regulated was not correlated with neither positive $(r=0.25, t(16)=1.03, p=0.32)$, nor negative $(r$ $=-0.39, t(16)=1.69, p=0.11)$ reappraisal scores.

A number of studies have provided evidence that effects of ER in the amygdala are associated with differential recruitment of the dorsolateral prefrontal cortex (DLPFC), a structure involved in cognitive control. To investigate this question, we correlated the observed reduction of activity within the bilateral amygdala (i.e., look - decrease trials) with the degree to which activity in the bilateral DLPFC was affected by ER. We removed subjects for which the effect of regulation within either the amygdala or DLPFC deviated by more than 2 standard deviations from the mean, which led to the removal of two healthy controls and one MDD patient for the correlation analysis for negative pictures. For healthy controls, the degree to which activity in the bilateral amygdala was downregulated to negative pictures was inversely correlated to activity in the bilateral DLPFC, $r=-0.54, t(14)=2.43, p=0.03$ (see Fig. 8). For MDD patients, there was no relationship between the effect of regulation on activity in the bilateral
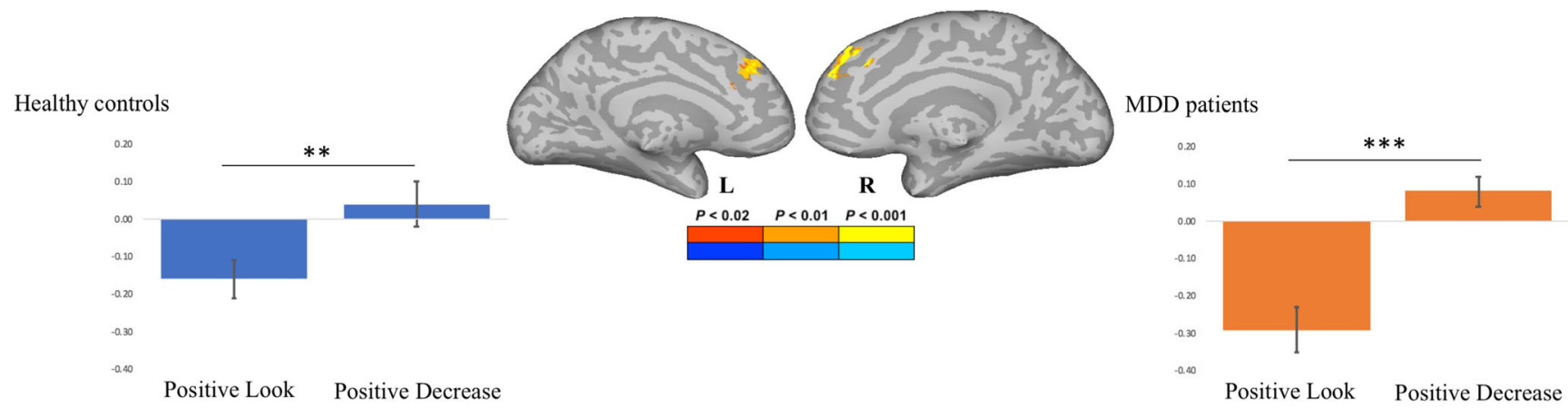

Fig. 10 Psycho-physiological interaction analysis showing that activity in the left dorsolateral prefrontal cortex was accompanied by taskdependent functional connectivity with the dorsomedial prefrontal cortex prior to stimulation. Parameter estimates of the psychophysiological

interaction analysis for the positive-look and positive-decrease condition for healthy controls and major depressive disorder (MDD) patients. $* *$ denotes $p<.01, * * *$ denotes $p<.001$ 


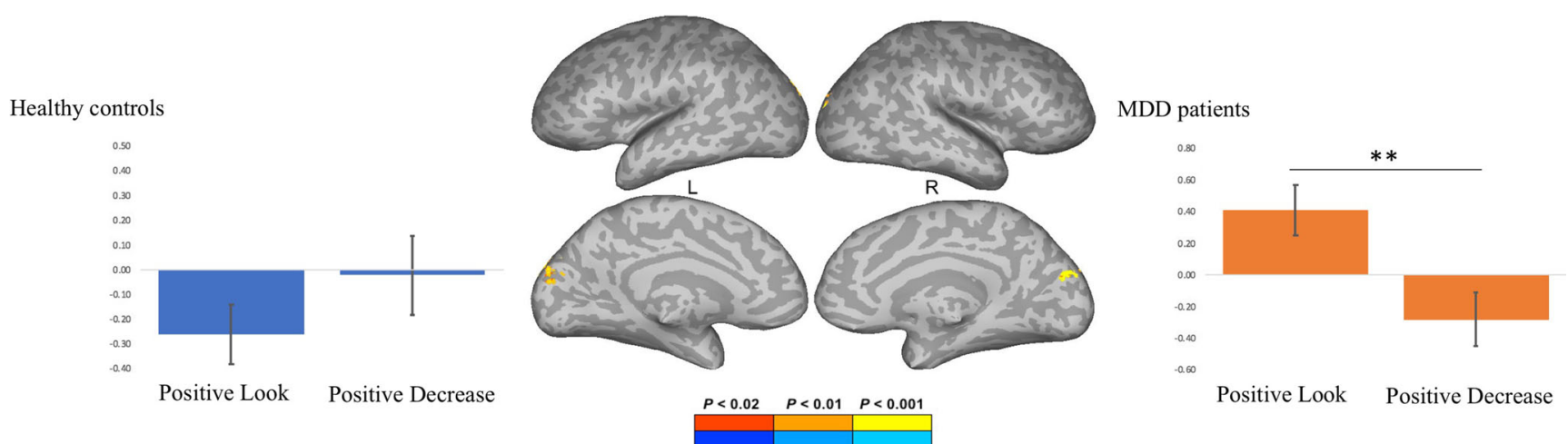

Fig. 11 Psycho-physiological interaction (PPI) analysis showing a differential PPI effect for healthy controls and major depressive disorder (MDD) patients in the left cuneus prior to stimulation. Parameter

amygdala and bilateral DLPFC, $r=-0.13, p>0.6$. No relationship was found between activity in the bilateral amygdala and bilateral DLPFC to positive pictures (all $p$ s $>0.3$ ).

\section{Psychophysiological interaction (PPI) analysis prior to stimulation}

The psychophysiological interaction (PPI) analysis showed that activity in the left dorsolateral prefrontal cortex (DLPFC) (physiological variable) was accompanied by taskdependent (negative decrease vs. negative look) functional connectivity with the cuneus, lingual gyrus, primary visual cortex, as well as the right IPL and AnG (Fig. 9). The ER $\times$ group interaction on the PPI term for the negative stimuli did not show any regions in which the PPI effect was different between MDD patients and control subjects. Plotting the parameter estimates of the psychophysiological interaction analysis for the negative-decrease and negative-look condition revealed that for both healthy controls and MDD patients the covariance in activity between the left DLPFC and the visual cortex was significantly higher during negative-decrease than during negative-look trials. Finally, we calculated the correlation coefficient between individual subjects' parameter estimates of the PPI term for the negative-decrease versus negative-look contrast and the participants' reappraisal scores. The Pearson's product-moment correlation between the parameter estimates of the PPI of the decrease versus look contrast and the negative reappraisal scores were not significant for either healthy controls $(r=0.04, t(16)=0.16, p>0.80)$ or MDD patients $(r=0.36, t(17)=1.59, p>0.13)$.

The psycho-physiological interaction (PPI) analysis showed that activity in the left DLPFC was accompanied by task-dependent (positive decrease vs. positive look) functional connectivity with the DMPFC (Fig. 10). Plotting the parameter estimates of the psychophysiological interaction analysis for the positive-decrease and positive-look condition revealed that for both controls and MDD patients, the covariance in activity between the left DLPFC and the DMPFC was higher estimates of the psychophysiological interaction analysis for the positive-look and positive-decrease condition for healthy controls and MDD patients. ** indicates $\mathrm{p}<.01$

during positive-decrease than during positive-look trials. The data from one healthy control were removed from the correlational analysis of the DMPFC and their reappraisal score, because it deviated more than 2 standard deviations from the mean. For healthy controls, the Pearson's product-moment correlation between the parameter estimates of the PPI of the decrease-look contrast and the positive reappraisal score did not reach statistical significance $(r=0.39, t(15)=1.66, p=$ 0.12 ). No relationship was observed between the parameters of the PPI effect and the positive reappraisal score for MDD patients $(r=0.13, t(17)=0.54, p>0.50)$.

The ER $\times$ group interaction on the PPI term from the positive stimuli showed a differential PPI effect between controls and patients within the left cuneus (Fig. 11). Plotting the parameter estimates of the psychophysiological interaction analysis for the positive-decrease and positive-look conditions revealed that healthy control subjects demonstrated increased left DLPFC-visual cortex coupling during ER, whereas patients demonstrated decreased left DLPFC-visual cortex coupling during ER. The Pearson's product-moment correlation between the parameter estimates from the PPI of the decreaselook contrast and the positive reappraisal scores were not significant for either healthy controls $(r=0.06, t(16)=0.24, p>$ $0.80)$ or MDD patients $(r=0.03, t(17)=0.12, p>0.90)$.

The PPI analysis in which we examined the psychophysiological interaction between the bilateral amygdala seed and the negative-decrease versus negative-look or positivedecrease versus positive-look contrast did not yield any significant results. $^{2}$

\footnotetext{
${ }^{2}$ The small difference in the number of males and females across the MDD and healthy control conditions might have influenced the results of some of the analyses. Specifically, a difference in ER between patients and control subjects could merely reflect a difference in the number of males in these groups rather than a difference in patient status. To ensure that our reported results reflect the latter (and not the former) possibility, we conducted all relevant analyses using gender as a covariate. For all of these analyses, the peak activation of each contrast was the same. According to these results, gender did not have any effect in modulating emotion regulation to either positive or negative pictures.
} 


\section{Effects of tDCS on ER networks}

The ER networks during tDCS stimulation (Negative/Positive Decrease vs. Negative/Positive Look contrast) across all subjects are presented in Supplementary Fig. 6 (Online Supplementary Material). To investigate the effect of active tDCS over left DLPFC on the neural networks involved in $\mathrm{ER}$, we ran a mixed-effect group analysis across all participant groups on the contrast (decrease-look during stimulation) (decrease-look pre-stimulation) with one fixed factor (stimulation: active vs. sham) and one random factor (participant), separately for negative and positive stimuli. The ER $\times$ tDCS stimulation interaction analysis for both the negative and the positive stimuli did not yield any statistically significant results. Furthermore, the three-way $($ ER $\times$ tDCS stimulation $\times$ group) interaction for negative and positive stimuli was not significant.

\section{Functional connectivity as a function of active versus sham tDCS}

The PPI analysis between the seed in either the left DLPFC (L-DLPFC) or bilateral amygdala (physiological variable) and the negative reappraisal during - negative reappraisal prestimulation contrast (the psychological variable) did not yield a differential PPI effect for participants who received active relative to sham tDCS stimulation. Similarly, the PPI analysis between the seed in either the L-DLPFC or bilateral amygdala and the positive reappraisal during - positive reappraisal prestimulation contrast did not yield a differential PPI effect for participants who received active relative to sham tDCS stimulation. Furthermore, in the above-mentioned analyses we did not find any significant Emotion regulation $\times$ tDCS Stimulation $\times$ Group interaction. That is, there was no evidence for a differential effect of tDCS stimulation on ER between MDD patients and healthy controls.

\section{Discussion}

The primary purpose of the present investigation was to examine the potential of unilateral excitatory tDCS over LDLPFC, with the reference electrode over the contralateral mastoid, to alter cortical excitability in a hypothesized deficient ER network in MDD patients relative to healthy control participants. We first discuss the results for the trials preceding the onset of stimulation, followed by a discussion of the results during tDCS.

\section{Pre-stimulation trials}

For the pre-stimulation trials, the whole-brain results revealed, in line with our predictions, an extensive network of regions implicated in ER across the posterior parietal and superior dorsal and middle lateral PFC. These results are consistent with prior work on the neural processing systems underlying ER: functional neuroimaging studies have provided evidence that cognitive reappraisal relies on a frontoparietal network including the DLPFC, ventral lateral prefrontal cortex (VLPFC), dorsomedial prefrontal cortex (DMPFC), and the dorsal anterior cingulate cortex (ACC) (McRae et al., 2010; Ochsner et al., 2004; Ochsner et al., 2002). It has been argued that these lateral and medial prefrontal areas implement control processes over the medial orbitofrontal cortex (MOFC) and the amygdala that encode and represent the affective properties of stimuli (Adolphs, 2002; Bechara et al., 1999; Rolls, 1999).

In line with this proposition, several neuroimaging studies have demonstrated that cognitive reappraisal is associated with a reduction of activity in the amygdala (Beauregard et al., 2001; Eippert et al., 2007; Ochsner et al., 2004; Phan et al., 2005). Our ROI analyses focused primarily on a prioridefined regions in the bilateral amygdala and the left DLPFC and showed stronger activation within the right amygdala when looking at negative pictures relative to when downregulating responses to these pictures in healthy controls (with the results for the left amygdala in the same direction, albeit not statistically significant following correction for multiple comparisons). In contrast, no differences were observed between the looking and ER trials for negative stimuli in MDD patients, in line with the anticipated difficulty in downregulation of negative emotional responses in that group. For positive stimuli, however, we observed decreased activity in the right amygdala when MDD patients attempted to downregulate their emotional responses to the positive images, relative to when they were simply looking at these images. We speculate that this difference might reflect MDD patients' tendency to reappraise positive emotional events in their lives, which consequently renders them more negative, thus presenting as the "negativity bias" often reported in the disorder. Coupled with the lack of differences in the down-regulation of activity in this region for negative stimuli for the MDD group and the presence of the reverse pattern of results for the healthy control group, we suggest that the significant down-regulation of positive but not negative events in the amygdala might be central to the neural presentation of depressive symptomatology (cf. Beblo et al., 2012).

We note that - although activity in the right amygdala was reduced during negative decrease relative to negative-look trials for healthy controls - the degree of down-regulation was positively correlated with the participants' reappraisal scores only within the left amygdala; no such relationship was observed with activity in the right amygdala. These findings are in line with a proposal put forth by Phelps et al. (2001) according to which laterality in amygdala activation in response to negative emotional stimuli may reflect the degree 
to which a stimulus depends on participants' explicit interpretation of and elaboration on the content of such stimuli. They argued that the right amygdala may be more strongly engaged when processing of the emotional properties of a stimulus is primarily visual, whereas the left amygdala activity may be observed when emotional properties of a stimulus are explicitly elaborated on verbally (for similar proposals pertaining to stimulus type, see Kensinger \& Schacter, 2006; Markowitsch, 1998; but see also Baas et al., 2004). Consistent with the proposal by Phelps et al. (2001), as participants in the present study were asked to explicitly reflect upon and evaluate via verbal labels the strength of their emotional response to the stimuli after each trial, their reappraisal scores correlated with the regulation of activity in the left, but not the right, amygdala.

Furthermore, our analysis revealed a novel finding for negative stimuli: There was a significant ER $\times$ group interaction within the lateral occipital cortex, cuneus, lingual gyrus, and the primary visual cortex, according to which ER trials were associated with reductions in activity in these regions for healthy participants, but not MDD patients. What's more, this down-regulation in visual cortex activity during ER for negative pictures was positively correlated with healthy participants' reappraisal scores during the task. These findings are in line with neuroimaging studies that have shown that mood disorders are associated with altered visual cortical and amygdala responses to emotional information with negative content (Anand et al., 2005; Furey et al., 2013; Lee et al., 2007). Specifically, similar to the inability of our MDD patients to effectively reduce activity within visual areas during ER, pretreatment neural activity within visual regions during emotional processing has been shown to predict who among MDD patients would respond favorably to scopolamine, further emphasizing the importance of the visual cortex in the processing of emotional information (Furey et al., 2013).

For healthy controls, the extent of bilateral amygdala down-regulation during ER trials for negative pictures was inversely correlated to activity in the bilateral DLPFC. These results are in line with previous findings demonstrating higher task-induced functional connectivity between the DLPFC, orbitofrontal cortex (OFC), ACC, DMPFC, inferior parietal cortex (IPC), and the amygdala when subjects reappraised as compared to maintained their emotions (Banks et al., 2007; Uchida et al., 2015). Consistent with past literature on the neurobiological mechanisms of MDD (e.g., DeRubeis et al., 2008; Disner et al., 2011), there was no relationship between the effect of regulation on activity in the bilateral amygdala and bilateral DLPFC in the patient group. These findings might reflect MDD patients' difficulties in disengaging attention away from negative emotional information due to a disrupted PFC function (Drevets, 2000; Leppänen, 2006; Mayberg, 2007) and/or reduced cortico-limbic connectivity in this group (Anand et al., 2005).
Lastly, a PPI analysis revealed that for both healthy control participants and MDD patients the covariance in activity between the left DLPFC and the visual cortex was significantly higher during decrease than during look trials for negative stimuli. However, for positive stimuli, we obtained a differential PPI effect within the visual cortex between MDD patients and healthy controls, evidenced by a significant ER $\times$ group interaction on the PPI term. That is, healthy control subjects demonstrated increased left DLPFC-visual cortex coupling during ER, whereas patients demonstrated decreased left DLPFC-visual cortex coupling during ER. These results are consistent with the finding that MDD patients differ with regard to the processing of positive stimuli, as reflected by greater suppression of positive emotions in the patients than in the healthy controls (Beblo et al., 2012). Furthermore, neuroimaging studies have shown that MDD patients show decreased activation in response to positive emotional stimuli in the right amygdala, rostral ACC, and medial frontal cortex (Kumari et al., 2003; Lee et al., 2007), with symptom severity being negatively correlated with rostral ACC activity (Lee et al., 2007). Although our study differed to an extent in its methodological details from this previous work, our findings from the pre-stimulation trials corroborate evidence that MDD patients are characterized by altered neural responses to positive emotional information. Furthermore, our results demonstrate decreased left DLPFC-visual cortex coupling of MDD patients during regulation of positive emotions, suggesting an increased ability of the LDLPFC of MDD patients to exert top-down regulatory control over the visual cortex during ER of positive emotions.

\section{Effects of tDCS}

Contrary to the outcomes of behavioral intervention studies with unilateral tDCS montages (Fitzgerald et al., 2013; Martin et al., 2011), our findings did not reveal significant behavioral or neural modulation effects as a result of anodal tDCS over left DLPFC using the present ER paradigm. Similarly, the PPI analysis between the seed in either the L-DLPFC or bilateral amygdala and either the negative or the positive reappraisal contrasts did not yield a differential PPI effect for participants who received active relative to sham tDCS stimulation or any differences between MDD patients and healthy control subjects. In contrast, we have recently shown (Chrysikou et al., in press) that a bilateral montage with active anodal tDCS over left DLPFC with concurrent cathodal tDCS over right DLPFC modulated activation in MPFC during reappraisal of negative stimuli; for MDD patients, but not control participants, this up-regulation of activity in the MPFC was predictive of reappraisal performance benefits from pre-stimulation to during stimulation trials, which we have shown might be attributed to the weakening of an aberrant functional connectivity between MPFC and the bilateral amygdala in MDD. 
In the context of this prior work, the present results support the conclusion that a unilateral montage with anodal tDCS over the left DLPFC (with the cathode electrode over the contralateral mastoid) does not appear to modulate significantly activation within a network involved in regulation of negative emotional stimuli in participants with MDD or healthy control subjects. With regard to the absence of significant tDCS effects, it could be argued that anodal tDCS over the left DLPFC affects both the "Look" and "Decrease" conditions, such that any potential effect of stimulation on ER is obscured by investigating the effect of tDCS on modulating the difference between conditions. A post hoc analysis of the effect of tDCS stimulation on Decrease and Look trials separately, revealed a significant Negative Look $\times$ stimulation interaction effect within the middle frontal gyrus (MFG; encompassing the ACC). No two- or three-way interaction effect was observed for the Negative-Decrease condition. These findings would suggest that the potential effects of tDCS on ER processes might be more complex and could be masked by traditional analyses of ER tasks that prioritize reappraisal scores.

Beyond task parameters, we note that the absence of significant tDCS effects in this experiment could be attributed to the specifics of the montage employed in the present study. For example, recent work has used current strengths up to $2.5 \mathrm{~mA}$ for $30 \mathrm{~min}$ (e.g., McClintock et al., 2020), whereas the present study employed $1.5 \mathrm{~mA}$ for $20 \mathrm{~min}$ to accommodate technical requirements of concurrent tDCS and fMRI at the time of data collection. It is possible that unilateral montages at higher current strengths or with increased focality as a result of smaller electrodes (i.e., high-definition tDCS) would elicit significant stimulation effects on this task. Lastly, we note that a potentially important limitation of the present investigation is the relatively small sample size per condition. Although our sample size was based on the limited prior work with concurrent tDCS and fMRI, it is possible that larger samples would allow for a more comprehensive investigation of tDCS effects over left DLPFC for ER in depression. In conjunction with our prior findings with a bilateral tDCS montage in a similar paradigm and with equivalent sample sizes, however (see Chrysikou et al., in press), the results of the present investigation likely point to a potential advantage of bilateral tDCS montages over PFC in the treatment of MDD.

\section{Conclusion}

The potential of tDCS as an effective treatment for MDD has been hampered by a lack of understanding of the precise effects of the procedure on the brain in the disorder that have further limited efforts to optimize tDCS parameters and maximize treatment outcomes. This study is among the handful of investigations that have leveraged fMRI-compatible tDCS technology to examine the consequences of tDCS for neural excitability in vivo in a group of moderate-to-severe MDD patients and age-matched control participants. Our results revealed a deficient ER network in MDD patients relative to control subjects, characterized by inefficiency in the downregulation of neural activity in response to negative stimuli within the bilateral amygdala and visual cortex. In the context of this ER paradigm and tDCS parameters, anodal tDCS over left DLPFC with the reference electrode away from the brain, did not elicit significant neuromodulation effects as measured by concurrent fMRI. Notwithstanding sample size considerations, these results provide support for the use of bilateral over unilateral tDCS montages as potential treatments for MDD.

Acknowledgements This work was supported by a CTSA grant from National Center for Advancing Translational Sciences (NCATS) awarded to the University of Kansas Medical Center for Frontiers: The Heartland Institute for Clinical and Translational Research (\# UL1TR000001). The Hoglund Brain Imaging Center is supported in part by a generous gift from Forrest and Sally Hoglund and an instrumentation grant from the National Institutes of Health; S10 RR29577. The contents are solely the responsibility of the authors and do not necessarily represent the official views of the NIH or NCATS or other funding agencies. We thank Gavin K. Hanson, Carla B. Fernandez, and W. Jake Thompson for assistance with portions of data collection.

Open practices statement Data, scripts, and materials for the experiments reported here are available upon request. The study was not preregistered.

\section{References}

Adolphs, R. (2002). Neural systems for recognizing emotion. Current Opinion in Neurobiology, 12, 169-177.

Anand, A., Li, Y., Wang, Y., Wu, J., Gao, S., Bukhari, L., et al. (2005). Activity and connectivity of brain mood regulating circuit in depression: a functional magnetic resonance study. Biological Psychiatry, 57, 1079-1088.

Antal A, Polania R, Schmidt-Samoa C, Dechent P, Paulus W (2011): Transcranial direct current stimulation over the primary motor cortex during fMRI. NeuroImage. 55: 590-596.

Arul-Anandam, A.P., Loo, C., 2009. Transcranial direct current stimulation: a new tool for the treatment of depression? Journal of Affective Disorders, 117, 137-145.

Baas, D., Aleman, A., \& Kahn, R. S. (2004). Lateralization of amygdala activation: a systematic review of functional neuroimaging studies. Brain Research Reviews, 45, 96-103.

Banks, S. J., Eddy, K. T., Angstadt, M., Nathan, P. J., \& Phan, K. L. (2007). Amygdala-frontal connectivity during ER. Social Cognitive and Affective Neuroscience, 2, 303-312.

Beauregard, M., Lévesque, J., \& Bourgouin, P. (2001). Neural correlates of conscious self-regulation of emotion. The Journal of Neuroscience, 21, 1-6.

Beblo, T., Fernando, S., Klocke, S., Griepenstroh, J., Aschenbrenner, S., \& Driessen, M. (2012). Increased suppression of negative and 
positive emotions in major depression. Journal of Affective Disorders, 141, 474- 479.

Bechara, A., Damasio, H., Damasio, A. R., \& Lee, G. P. (1999). Different contributions of the human amygdala and ventromedial prefrontal cortex to decision-making. Journal of Neuroscience, 19, 54735481.

Benjamini, B. \& Hochberg, Y. (1995). Controlling the false discovery rate: a practical and powerful approach to multiple testing. Journal of the Royal Statistical Society, Series B 57 (1), 289-300.

Bergmann TO, Karabanov A, Hartwigsen G, Thielscher A, Siebner HR (2016): Combining non-invasive transcranial brain stimulation with neuroimaging and electrophysiology: Current approaches and future perspectives. NeuroImage. 140: 4-19.

Berlim, M.T., Van den Eynde, F., Tovar-Perdomo, S., Chachamovich, E., Zangen, A., Turecki, G., 2014. Augmenting antidepressants with deep transcranial magnetic sti-mulation (DTMS) in treatmentresistant major depression. World Journal of Biological Psychiatry, 15 (7), 570-578.

Bikson, M., Bulow, P., Stiller, J.W., Datta, A., Battaglia, F., Karnup, S.V., Postolache, T.T., 2008. Transcranial direct current stimulation for major depression: a general system for quantifying transcranial electrotherapy dosage. Current Treatment Options in Neurology 10, 377-385.

Bikson, M., Grossman, P., Thomas, C., Zannou, A. L., Jiang, J., Adnan, T., et al. (2016) Safety of transcranial direct current stimulation: evidence based update 2016. Brain Stimulation, 9, 641-661. https://doi.org/10.1016/j.brs.2016.06.004

Boggio, P. S. Rigonatti S.P., Ribeiro, R.B., Myczkowski, M.L., Nitsche, M.A., Pascual-Leone, A., \& Fregni, F. (2008). A randomized, double-blind clinical trial on the efficacy of cortical direct current stimulation for the treatment of major depression. The International Journal of Neuropsychopharmacology, 11, 249-254.

Breiter, H. C., Etcoff, N. L., Whalen, P. J., Kennedy, W. A., Rauch, S. L., Buckner, R. L., Strauss, M. M., Hyman, S. E., \& Rosen, B. R. (1996). Response and habituation of the human amygdala during visual processing of facial expression. Neuron, 17, 875-887.

Brunoni AR, Moffa AH, Fregni F, Palm U, Padberg F, Blumberger DM, et al. (2016): Transcranial direct current stimulation for acute major depressive episodes: meta-analysis of individual patient data. The British Journal of Psychiatry 208: 522-531.

Bush, G., Luu, P., and Posner, M. (2000). Cognitive and emotional influences in anterior cingulate cortex. Trends in Cognitive Sciences, 4, 215-222.

Chase HW, Boudewyn MA, Carter CS, Phillips ML (2019): Transcranial direct current stimulation: a roadmap for research, from mechanism of action to clinical implementation. Molecular Psychiatry 1-11.

Chrysikou EG, Hanson GK, Ramey CH, Martin LE, Choi I-Y, Lee P, Brooks WM (2017): Transcranial direct current stimulation effects on prefrontal cortex in major depressive disorder (MDD) measured by in vivo proton magnetic resonance spectroscopy ( ${ }^{1} \mathrm{H}$ MRS). Brain Stimulation, 10, e53-e54.

Chrysikou, E. G., Wing, E. K., \& van Dam, W. O., (in press). Transcranial direct current stimulation over prefrontal cortex in depression modulates cortical excitability in ER regions as measured by concurrent functional magnetic resonance imaging: An exploratory study. Biological Psychiatry: CCNI

Cowden Hindash, A, Amir, N (2012) Negative interpretation bias in individuals with depressive symptoms. Cognitive Therapy and Research, 36, 502-511.

Cox, R. W. (1996). AFNI: Software for analysis and visualization of functional magnetic resonance neuroimages. Computers and Biomedical Research, 29: 162-173.

Cox, R. W., \& Jesmanowicz A (1999). Real-time 3D image registration of functional MRI. Magnetic Resonance in Medicine, 42, 1014 1018 .
Cox, R. W., Reynolds, R. C., \& Taylor, P. A. (2016). AFNI and clustering: false positives rates redux. bioRxiv 065862. https://doi.org/10. 1101/065862.

DeRubeis RJR, Siegle GJG, Hollon SDS (2008): Cognitive therapy versus medication for depression: treatment outcomes and neural mechanisms. Nature Reviews. Neuroscience 9: 788-796.

Desikan, R. S., Ségonne, F., Fischl, B., Quinn, B. T., Dickerson, B.C., Blacker, D., ... Killiany, R. J. (2006). An automated labeling system for subdividing the human cerebral cortex on MRI scans into gyral based regions of interest. Neuroimage, 31(3), 968-980.

Disner, S. G., Beevers, C. G., Haigh, E. A. P., \& Beck, A. T. (2011). Neural mechanisms of the cognitive model of depression. Nature Reviews Neuroscience, 12(8), 467e477. https://doi.org/10.1038/ nrn3027.

Dmochowski JP, Datta A, Bikson M, Su Y, Parra LC (2011): Optimized multi-electrode stimulation increases focality and intensity at target. Journal of Neural Engineering 8: 046011-17.

Drevets, W. C. (2000). Neuroimaging studies of mood disorders. Biological Psychiatry, 48, 813-829.

Eippert, F., Veit, R., Weiskopf, N., Erb, M., Birbaumer, N., \& Anders, S. (2007). Regulation of emotional responses elicited by threat-related stimuli. Human Brain Mapping, 28(5), 409-423.

Fitzgerald, P. B., Hoy, K. E., Singh, A., Gunewardene, R., Slack, C., Ibrahim, S., Hall, P.J., \& Daskalakis, Z.J. (2013). Equivalent beneficial effects of unilateral and bilateral prefrontal cortex transcranial magnetic stimulation in a large randomized trial in treatmentresistant major depression. The International Journal of Neuropsychopharmacology, 16, 1975-1984. doi:https://doi.org/10. 1017/S1461145713000369

Fischl, B. (2012) FreeSurfer. NeuroImage, 62(2),774-781

Flöel A (2012): Non-invasive brain stimulation and language processing in the healthy brain. Aphasiology, 26, 1082-1102.

Fregni F, Boggio PS, Nitsche MA, Rigonatti SP, Pascual-Leone A (2006): Cognitive effects of repeated sessions of transcranial direct current stimulation in patients with depression. Depression and Anxiety 23: 482-484.

Friston, K. J. (1994). Functional and effective connectivity in neuroimaging: a synthesis. Human Brain Mapping, 2, 56-78.

Furey ML, Drevets WC, Hoffman EM, Frankel E, Speer AM, Zarate, CA $\mathrm{Jr}$ (2013). Potential of pretreatment neural activity in the visual cortex during emotional processing to predict treatment response to scopolamine in major depressive disorder. JAMA Psychiatry; 70: 280-290.

Giordano, J., Bikson, M., Kappenman, E.S., Clark, V.P., Coslett, B., Hamblin, M.R., et al. (2017). Mechanisms and effects of transcranial direct current stimulation. Dose-Response, 5, 1-22, doi: https://doi. org/10.1177/1559325816685467.

Greve, D. N. (2002). Optseq Home Page. Available online at http://surfer. nmr.mgh.harvard.edu/optseq

Gross, J. J. (1998). Antecedent- and response-focused ER: divergent consequences for experience, expression, and physiology. Journal of Personality and Social Psychology, 74, 224-237.

Gross, J. J., \& John, O. P. (2003). Individual differences in two ER processes: implications for affect, relationships, and well-being. Journal of Personality and Social Psychology, 85(2), 348-362.

Gross, J. J., \& Levenson, R. W. (1997). Hiding feelings: the acute effects of inhibiting positive and negative emotions. Journal of Abnormal Psychology, 106, 95-103.

Hunter MA, Coffman BA, Trumbo MC (2013): Tracking the neuroplastic changes associated with transcranial direct current stimulation: a push for multimodal imaging. Frontiers in Human Neuroscience. https://doi.org/10.3389/fnhum.2013.00495/full.

Jamil, A., Batsikadze, G., Kuo, H.I., Labruna, L., Hasan, A., Paulus, W., et al. (2017). Systematic evaluation of the impact of stimulation intensity on neuroplastic after-effects induced by transcranial direct 
current stimulation. The Journal of Physiology, 595, 1273-1288. doi: https://doi.org/10.1113/JP272738.

Janicak, P. G., \& Dokucu, M. E. (2015). Transcranial magnetic stimulation for the treatment of major depression. Neuropsychaitric Disease and Treatment, 11, 1549-1560.

John, O. P., \& Gross, J. J. (2004). Healthy and unhealthy ER: personality processes, individual differences, and life span development. Journal of Personality, 72(6), 1301-1334.

Joorman, J., \& Stanton, C. H. (2016). Examining ER in depression: A review and future directions. Behaviour Research and Therapy, 86, $35-49$.

Joorman, J., \& Vanderlind, M. (2014). Emotion regulation in depression: The role of biased cognition and reduced cognitive control. Clinical Psychological Science, 2, 402-421.

Joormann, J., \& Gotlib, I. H. (2010). Emotion regulation in depression: Relation to cognitive inhibition. Cognition \& Emotion, 24(2), 281e298. https://doi.org/10.1080/02699930903407948.

Kensinger, E. A., \& Schacter, D. L. (2006). Processing emotional pictures and words: effects of valence and arousal. Cognitive, Affective, \& Behavioral Neuroscience, 6(2), 110-126.

Kessler, R. C. (2012). The costs of depression. Psychiatric Clinics of North America, 35, 1-14. doi:https://doi.org/10.1016/j.psc.2011. 11.005

Kessler, R. C., \& Bromet, E. J. (2013). The epidemiology of depression across cultures. Annual Review of Public Health, 9, 119-138. doi: https://doi.org/10.1146/annurev-publhealth-031912-114409

Kronberg, G., Bridi, M., Abel, T., Bikson, M., \& Parra, L. C. (2017). Direct current stimulation modulates LTP and LTD: Activity dependence and dendritic effects. Brain Stimulation, 10, 51-58. doi: https://doi.org/10.1016/j.brs.2016.10.001.

Kumari, V., Mitterschiffthaler, M. T., Teasdale, J. D., Malhi, G. S., Brown, V., Giampietro et al. (2003). Neural abnormalities during cognitive generation of affect in treatment-resistant depression. Biological Psychiatry, 54, 777-791.

Lang, P.J., Bradley, M.M., \& Cuthbert, B.N. (2008). International affective picture system (IAPS): Affective ratings of pictures and instruction manual. Technical Report A-8. University of Florida, Gainesville, FL.

Lazarus, R. S., \& Alfert, E. (1964). Short-circuiting of threat by experimentally altering cognitive appraisal. Journal of Abnormal and Social Psychology, 69, 195-205.

Lee, B-T., Cho, S. W., Khang, H. S., Lee, B-C, Choi, I-G., Lyoo, I. K., \& Ham, B-J. (2007). The neural substrates of affective processing toward positive and negative affective pictures in patients with major depressive disorder. Progress in Neuro-Psychopharmacology and Biological Psychiatry, 31(7), 1487-1492.

Lee, H., Heller, A. S., van Reekum, C. M., Nelson, B., \& Davidson, R. J. (2012). Amygdala-prefrontal coupling underlies individual differences in ER. Neuroimage, 62, 1575-1581.

Leppänen, J. M. (2006). Emotional information processing in mood disorders: a review of behavioral and neuroimaging findings. Current Opinion in Psychiatry, 19, 34-39.

Liverant, G. I., Brown, T. A., Barlow, D. H., \& Roemer, L. (2008). Emotion regulation in unipolar depression: The effects of acceptance and suppression of subjective emotional experience on the intensity and duration of sadness and negative affect. Behaviour Research and Therapy, 46(11), 1201e1209. https://doi.org/10. 1016/j.brat.2008.08.001.

Liverant, G. I., Kamholz, B. W., Sloan, D. M., \& Brown, T. A. (2011). Rumination in clinical depression: A type of emotional suppression? Cognitive Therapy and Research, 35, 253-265. https://doi.org/10. 1007/s10608-010-9304-4

Markowitsch, H. J. (1998). Differential contribution of right and left amygdala to affective information processing. Behavioral Neurology, 11, 233-244.
Martin, D. M., Alonzo, A., Mitchell, P. B., Sachdev, P., Gálvez, V., \& Loo, C. K. (2011). Fronto-extracephalic transcranial direct current stimulation as a treatment for major depression: An open-label pilot study. Journal of Affective Disorders, 134(1-3), 459-463. https:// doi.org/10.1016/j.jad.2011.05.018

Mayberg, H. S. (2007). Defining the neural circuitry of depression: toward a new nosology with therapeutic implications. Biological Psychiatry, 61, 729-730.

McClintock SM, Martin DM, Lisanby SH, Alonzo A, McDonald WM, Aaronson ST, Husain MM, O'Reardon JP, Weickert CS, Mohan A, Loo CK. (2020). Neurocognitive effects of transcranial direct current stimulation (tDCS) in unipolar and bipolar depression: Findings from an international randomized controlled trial. Depression and Anxiety, 1-12.

McLaren, D. G., Ries, M. L., Xu, G., Johnson, S. C. (2012). A generalized form of context-dependent psychophysiological interactions (gPPI): a comparison to standard approaches. NeuroImage, 61(4), 1277-1286. doi:https://doi.org/10.1016/j.neuroimage.2012.03.068.

McRae, K., Hughes, B., Chopra, S., Gabrieli, J. D. E., Gross, J. J., \& Ochsner, K. N. (2010). The neural bases of distraction and reappraisal. Journal of Cognitive Neuroscience, 22(2), 248-262.

Meinzer M, Antonenko D, Lindenberg R, Hetzer S, Ulm L, Avirame K, et al. (2012): Electrical Brain Stimulation Improves Cognitive Performance by Modulating Functional Connectivity and TaskSpecific Activation. The Journal of Neuroscience 32: 1859-1866.

Miranda, P.C., Lomarev, M., Hallett, M., 2006. Modeling the current distribution during transcranial direct current stimulation. Clinical Neurophysiology 117, 1623-1629.

Mitterschiffthaler, M. T., Williams, S. C., Walsh, N. D., Cleare, A., Donaldson, C., Scott, J., \& Fu, C. H. (2008). Neural basis of the emotional Stroop interference effect in major depression. Psychological Medicine, 38, 247-256.

Morris, J. S., Frith, C. D., Perrett, D. I., Rowland, D., Young, A. W., Calder, A. J., \& Dolan, R. J. (1996). A differential neural response in the human amygdala to fearful and happy facial expressions. Nature, 383, 812-815.

Nitsche, M. A., \& Paulus, W. (2000). Excitability changes induced in the humanmotor cortex by weak transcranial direct current stimulation. Journal of Physiology, 527, 633-639.

Nitsche, M. A., \& Paulus, W. (2001). Sustained excitability elevations induced by transcranial DC motor cortex stimulation in humans. Neurology, 57(10), 1899-1901.

O'Reilly, J. X., Woolrich, M. W., Behrens, T. E. J., Smith, S. M., Johansen-Berg, H. (2012). Tools of the trade: psychophysiological interactions and functional connectivity. Social Cognitive and Affective Neuroscience, 7(5), 604-609.

Ochsner, K. N., Bunge, S. A., Gross, J. J., \& Gabrieli, J. D. E. (2002). Rethinking feelings: an fMRI study of the cognitive regulation of emotion. Journal of Cognitive Neuroscience, 14(8), 1215-1229.

Ochsner, K. N., Ray, R. D., Cooper, J. C., Robertson, E. R., Chopra, S., Gabrieli, J. D., \& Gross, J. J. (2004). For better or for worse: neural systems supporting the cognitive down- and up-regulation of negative emotion. Neuroimage, 23, 483-499.

Ochsner, K. N., Silvers, J. A., \& Buhle, J. T. (2012). Functional imaging studies of ER: a synthetic review and evolving model of the cognitive control of emotion. Annals of the New York Academy of Sciences, https://doi.org/10.1111/j.1749-6632.2012.06751.x

Shiozawa, P., Fregni, F., Benseñor, IM., Lotufo, PA., Berlim, MT., Daskalakis, JZ., Cordeiro, Q., Brunoni, A. R. (2014) Transcranial direct current stimulation for major depression: an updated systematic review and meta-analysis. The International Journal of Neuropsychopharmacology, 17(09):1443-1452.

Phan, K. L., Fitzgerald, D. A., Nathan, P. J., Moore, G. J., Uhde, T. W., \& Tancer, M. E. (2005). Neural substrates for voluntary suppression of negative affect: a functional magnetic resonance imaging study. Biological Psychiatry, 57(3), 210-219. 
Phelps, E. A., O’Connor, K. J., Gatenby, C., Gore, J. C., Grillon, C., \& Davis, M. (2001). Activation of the left amygdala to a cognitive representation of fear. Nature Neuroscience, 4(4), 437-441.

Purpura, D. P., \& McMurtry, J. G. (1965). Intracellular activities and evoked potential changes during polarization of motor cortex. Journal of Neurophysiology, 28, 166-185.

Rolls, E. T. (1999). The brain and emotion. New York: Oxford University Press.

Rush, A. J., Trivedi, M. H., Wisniewski, S. R., Stewart, J. W., Nierenberg, A.A., Thase, M. E., ... Fava, M. (2006). BupropionSR, Sertraline, or Venlafaxine-XR after Failure of SSRIs for depression. New England Journal of Medicine, 354(12), 1231-1242. https://doi.org/10.1056/NEJMoa052963

Saad, Z. S., Glen, D. R., Chen, G., Beauchamp, M. S., Desai, R., \& Cox, R. W. (2009). A new method for improving functional-to-structural MRI alignment using local Pearson correlation. Neuroimage, 44, 839-848.

Samani, M. M., Agboada, D., Jamil, A., Kuo, M.-F., \& Nitsche, M. A. (2019). Titrating the neuroplastic effects of cathodal transcranial direct current stimulation (tDCS) over the primary motor cortex. Cortex, 1-29. https://doi.org/10.1016/j.cortex.2019.04.016

Sehm B, Schafer A, Kipping J, Margulies D, Conde V, Taubert M, et al. (2012): Dynamic modulation of intrinsic functional connectivity by transcranial direct current stimulation. Journal of Neurophysiology 108: 3253-3263.
Talairach J., \& Tournoux, P. (1988). Co-planar stereotaxic atlas of the human brain. New York: Thieme Medical.

Turi Z, Paulus W, Antal A (2012): Functional Neuroimaging and Transcranial Electrical Stimulation. Clinical EEG and Neuroscience 43: 200-208.

Uchida, M., Biederman, J., Gabrieli, J. D. E., Micco, J., de Los Angeles, C., Brown, A., Kenworthy, T., Kagan, \& Whitfield-Gabrieli, S. (2015). Emotion regulation ability varies in relation to intrinsic functional brain architecture. Social Cognitive and Affective Neuroscience, 1738-1748.

Van Essen, D. C., Drury, H. A., Dickson, J., Harwell, J., Hanlon, D., \& Anderson, C. H. (2001). An integrated software suite for surfacebased analyses of cerebral cortex. Journal of the American Medical Informatics Association: JAMIA, 8(5), 443-459.

Wager, T. D., Davidson, M. L., Hughes, B. L., Lindquist, M. A., \& Ochsner, K. N. (2008). Prefrontal-subcortical pathways mediating successful ER. Neuron, 59, 1037-1050.

World Health Organization, 2018. Depression. Available from. www. who.int/mediacentre/factsheets/fs369/en/ (accessed 11 November 2019).

Publisher's note Springer Nature remains neutral with regard to jurisdictional claims in published maps and institutional affiliations. 